Research Article

\title{
Shaking Table Tests for Seismic Response of Orthogonal Overlapped Tunnel under Horizontal Seismic Loading
}

\author{
Honggang Wu $\left(\mathbb{D},{ }^{1}\right.$ Hao Lei $\mathbb{D}^{1},{ }^{1,2}$ and Tianwen Lai ${ }^{2}$ \\ ${ }^{1}$ Northwest Research Institute Co., Ltd of C.R.E.C, Lanzhou, Gansu 730000, China \\ ${ }^{2}$ School of Civil Engineering, Lanzhou Jiaotong University, Lanzhou, Gansu 730000, China \\ Correspondence should be addressed to Hao Lei; 714664532@qq.com
}

Received 19 November 2020; Revised 29 December 2020; Accepted 30 December 2020; Published 19 January 2021

Academic Editor: Ma Jianjun

Copyright (C) 2021 Honggang Wu et al. This is an open access article distributed under the Creative Commons Attribution License, which permits unrestricted use, distribution, and reproduction in any medium, provided the original work is properly cited.

This paper presents the seismic dynamic response and spectrum characteristics of an orthogonal overlapped tunnel by shaking table tests. First, a prototype of the engineering and shaking table test device, which was used to design details of the experiment, was developed. Then, the sensors used in the test were selected, and the measurement points were arranged. Subsequently, the Wenchuan seismic wave with horizontal direction in different peak ground accelerations was inputted into the model, followed by a short analysis of the seismic response of the overlapped tunnel in the shaking table test as well as the distribution of the peak acceleration. Throughout the studies, the model exhibited obvious deformation stages during the seismic wave loading process, which can be divided into elastic, plastic, plastic enhancement, and failure stage. In particular, the time- and frequency-domain characteristics of the key parts of the tunnel were discussed in detail by using the continuous wavelet transform (CWT) based on the Morlet wavelet as the basis function. We found that the acceleration response was more intense within 25-60 s after the seismic wave was inputted. Furthermore, owing to "the superposition effect," the seismic response at the crown of the under-crossing tunnel was stronger than that at the invert of the upper-span tunnel. The low and medium frequencies in the transformation of small scales (5-20) significantly affected the overlapped tunnel. These results elucidate the seismic dynamic response of the overlapped tunnel and provide guidance for the design of stabilizing structures for reinforcing tunnels against earthquakes.

\section{Introduction}

With the development and utilization of underground space, three-dimensional intersections or close constructions between tunnels inevitably occur [1-3]. However, the overlapped tunnel, which differs from the conventional single tunnel, is relatively complex under the action of seismic loading because of its relatively ambiguous load and sensitivity to the surrounding environment. Additionally, owing to their unique features, overlapped tunnels are often subjected to the interactions of multiple seismic waves such as incident, reflection, and diffraction waves simultaneously under the action of seismic loading [4-6]. Hence, it is important to study the dynamic response of the overlapped tunnel under seismic loading.

In general, theoretical analysis, numerical analysis, and physical modelling are commonly used for evaluating the seismic performance of underground structures, which have been studied by many researchers. The pioneering study in the theoretical analysis of the seismic response of the tunnel was performed by St. John and Zahrah [7], and their theory is still used in subsequent studies [8-11]. They presented an advanced review of the current understanding of the seismic behavior of tunnels. According to their theory, the seismic performance and damage characteristics of tunnel structures differ from those of aboveground structures. Numerical analysis has also been used to examine the mechanical and deformation characteristics of tunnels under seismic loading [12-15]. Through numerical analysis, the tunnel response can be simulated, and the results can be compared with model test and field test results, which is one of the most used methods for seismic response analysis in tunnel engineering. Additionally, numerical simulations of the seismic response of double-deck overlapped tunnels of subways 
were performed by Jiang et al. [16] and Wang et al. [17] who focused on the effects of the cross form and input seismic waves on the seismic response characteristics of the subway tunnel.

Physical modelling is an important method for evaluating the earthquake resistance of underground structures $[18,19]$. Generally, a shaking table test is used, which can reproduce recorded ground motions or input artificial seismic waves. This is the most direct way to study the dynamic characteristics of structures, failure mechanisms, and seismic measures. For instance, Wang et al. [20] and Jiang et al. [21] performed shaking table tests to study the seismic performance of space-parallel overlapped tunnels under different seismic waves, loading directions, and amplitudes. The results indicated that the amplification coefficient of acceleration in $Z$ direction was generally larger than that in $X$ direction, and the seismic responses at different sections of the tunnel were significantly different. Chen et al. [22] and Zhao et al. [23] studied the overlapped subway tunnel by using the gypsum tunnel model and found that the peak strain and degree of damage on both sides of the tunnel model were distributed in the shape of an "S" along the height direction. Additionally, the seismic response exhibited a significant spatial effect in all parts of the tunnel. This conclusion was also drawn by Kawamata et al. [24] and Wang et al. [25] in experimental studies. Furthermore, MasoudRabeti et al. [26] examined the influence of the circular tunnel on the amplification mode of ground motion and analyzed the effects of the shear wave velocity of the soil, input motion frequency, flexibility ratio, tunnel depth, and other parameters on the amplification mode. Liu et al. [27] carried out a large-scale shaking table test to study the seismic response of crossing tunnel, and the acceleration and strain showed different dynamic responses, which were verified by numerical analysis subsequently.

Although scholars have conducted numerous studies, our understanding of the dynamic characteristics of the overlapped tunnel, as well as their variation with the application of seismic loads, remains poor. In some cases, the studies focused on a single-line tunnel or metro shield tunnel, and the research methods mainly involved theoretical analysis and numerical simulation. Research on the overlapped tunnel has not achieved substantial breakthroughs, and the related research results are rarely published. Therefore, to elucidate the seismic dynamic response of the overlapped tunnel and improve the reliability of the dynamic parameters of overlapped structures, a series of shaking table tests were performed for the overlapped tunnel. The remainder of this paper is organized as follows. First, details regarding the model and similar materials are presented, including the similarity ratios, dimensions of the model box, model parameters, and surrounding rock materials. The subsequent sections describe the sensors included in the tests, the arrangement of the measurement points, and the direction and peak ground acceleration (PGA) of the input seismic waves. Furthermore, the shaking table test results are presented in detail, and the time- and frequency-domain characteristics of the key parts of the tunnel are discussed. Finally, the seismic responses and seismic properties of the overlapped tunnel are summarized.

\section{Experiment Setup}

2.1. Engineering Prototype. Strawberry ditch tunnel No. 2 is a single-hole and double-line tunnel with a total length of $4262 \mathrm{~m}$ and a buried depth of 20-150 m. The total length of the Pandaoling Highway tunnel is $620 \mathrm{~m}$, with a buried depth of 17.5-106.5 m. Strawberry ditch tunnel No. 2 underpasses the Pandaoling Highway tunnel (hereinafter referred to as the upper-span and the under-crossing tunnels, respectively) at TJLDK $158+161$, with a clear structure distance of only $7.47 \mathrm{~m}$. This is a spatial orthogonal overlapped tunnel, as shown in Figure 1.

In Figure 1, the red lines on the bottom and left represent Strawberry ditch tunnels No. 1 and No. 2, respectively; the red line on the right represents Pandaoling tunnel; the blue line represents Jinjiangshan tunnel; the purple lines on the top represent the left and right lines of Pandaoling Highway tunnel; and the arrow indicates the main research object of this paper; namely, Strawberry ditch tunnel No. 2 underpasses the left line of Pandaoling Highway tunnel.

We used a TRT6000 advanced geological detector to forecast the geological conditions in the cross-affected zone. The surrounding rock was mainly composed of mixed granite with different degrees of weathering and developed joint fissures, and the rock mass was massive and fragmentary, with $V_{s}$ of $1000 \mathrm{~m} / \mathrm{s}$ and $V_{p}$ of $2600 \mathrm{~m} / \mathrm{s}$. The crossing section where the tunnel was located had a varied topography and belonged to a hilly geomorphic unit. The maximum elevation of the mountaintop was approximately $154 \mathrm{~m}$, and the maximum freezing depth of soil in the crossing section was $1.2 \mathrm{~m}$. According to the relevant Chinese criterion "Code for Design of Railway Tunnels (TB 10003-2016)" and "Earthquake Parameter Zoning Map of China (2015) (GB18306)," the surrounding rock condition was set as grade IV, and the site area corresponded to the basic seismic intensity of grade VII, which might have caused destructive earthquakes.

2.2. Similarity Ratios. Generally, for studying the seismic responses of tunnels, gravity distortion models are often used, because of the difficulty of adding additional weights [28]. Considering the dimensions of the model box and the depth of the tunnel, the following basic parameters were used: the geometric dimension $L\left(C_{L}=1 / 50\right)$, elastic modulus $E\left(C_{E}=1 / 30\right)$, and density $\rho\left(C_{\rho}=1 / 1\right)$. The similarity ratios of other physical parameters were derived using the Buckingham $\pi$ theorem [29], as shown in Table 1.

\subsection{Similar Materials of Surrounding Rock and Lining.} Owing to the complexity of the material properties of the surrounding rock, it is difficult for the dynamic model tests to fully comply with the similarity theorem. For the selection of the surrounding rock materials, the physical and mechanical parameters that affected the seismic response were mainly considered: the bulk density, cohesion, internal 


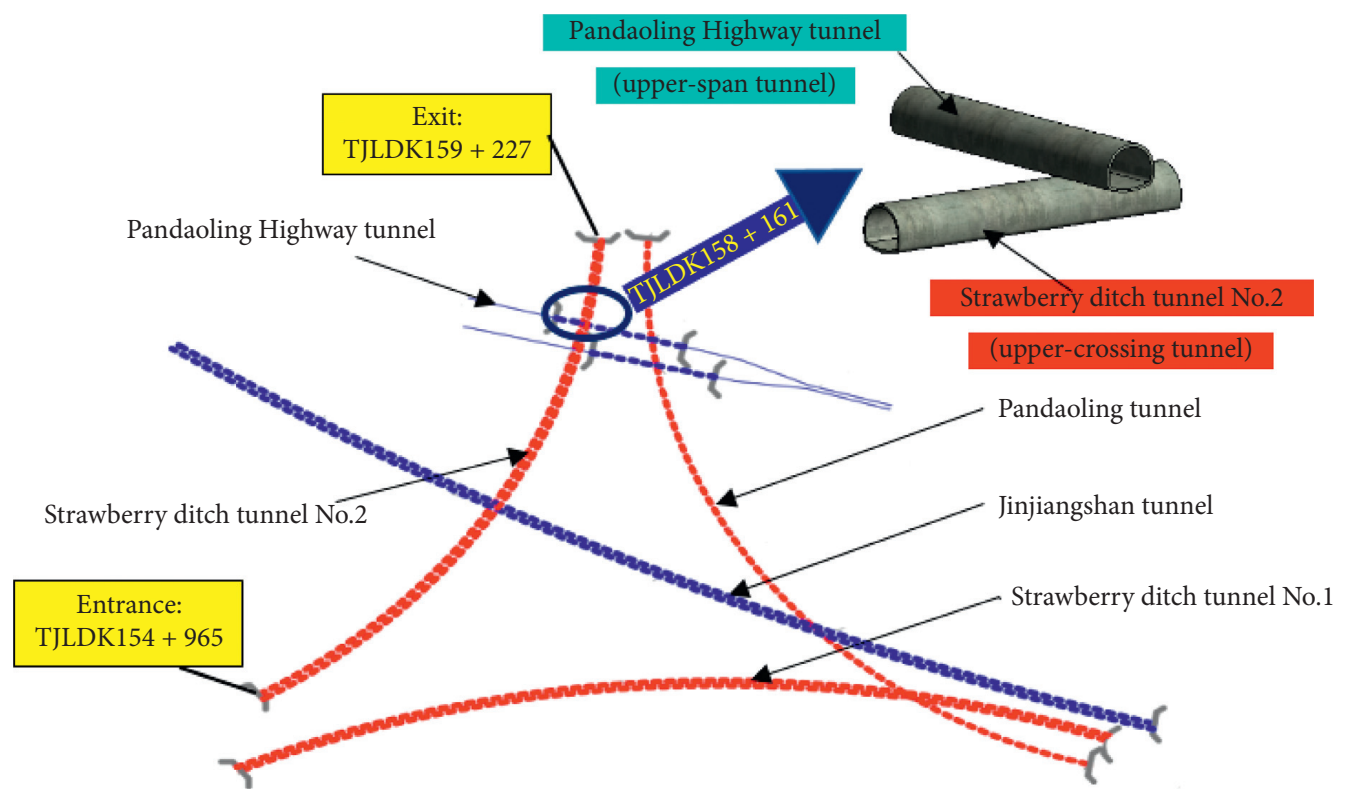

FIgURE 1: The spatial location of the overlapped tunnel.

TABLE 1: Similarity relations and similarity constants.

\begin{tabular}{|c|c|c|c|c|}
\hline Model composition & & Physical parameters & Similarity relations & Similarity constants \\
\hline \multirow{3}{*}{\multicolumn{2}{|c|}{ Basic parameters }} & Length $(L)$ & $C_{l}$ & $1 / 50$ \\
\hline & & Density $(\rho)$ & $C_{\rho}$ & $1 / 1$ \\
\hline & & Elastic modulus $(E)$ & $C_{E}$ & $1 / 30$ \\
\hline \multirow{8}{*}{ Derived parameters } & \multirow{4}{*}{ Model material parameters } & Stress $(\sigma)$ & $C_{\sigma}=C_{E} C_{\varepsilon}$ & $1 / 30$ \\
\hline & & Strain $(\varepsilon)$ & $C_{\varepsilon}=1$ & $1 / 1$ \\
\hline & & Poisson's ratio $(\mu)$ & $C_{\mu}=1$ & $1 / 1$ \\
\hline & & Internal friction angle $(\phi)$ & $C_{\phi}=1$ & $1 / 1$ \\
\hline & \multirow{4}{*}{ Seismic response parameters } & Time $(t)$ & $C_{t}=C_{\rho}^{0.5} C_{E}^{-0.5} C_{L}$ & $1 / 9.129$ \\
\hline & & Displacement $(d)$ & $C_{d}=C_{L}$ & $1 / 50$ \\
\hline & & Velocity $(v)$ & $C_{v}=C_{\rho}^{0.5} C_{E}^{-0.5}$ & $1 / 0.183$ \\
\hline & & Acceleration $(a)$ & $C_{a}=C_{\rho}^{-1} C_{E} C_{L}^{-1}$ & $1 / 0.6$ \\
\hline
\end{tabular}

friction angle, elastic modulus, and so forth. To improve efficiency, orthogonal design is used to set the comparison group test; that is, only a single parameter is changed each time. The physical and mechanical parameters are determined by direct shear test and triaxial test (Figure 2) on similar materials with different mix ratios, which are presented in Table 2.

Finally, a mixture of cement, coarse sand, soil, and water at a weight ratio of $0.5: 12: 5: 2$ was used to simulate the surrounding rock. The lining structure of the tunnel was made of a mixture of gypsum, quartz sand, and water, with a ratio of $1: 1.5: 2$. This method of mixing the surrounding rock has also been used in previous studies, and its effectiveness has been validated [30,31].

2.4. Design of the Model. In this study, the overlapped tunnels were selected as the reference model, and the tests were performed using the servo-driven seismic simulation shake table system of the Lanzhou Institute of Seismology of the China Earthquake Administration [32]. The relevant parameters of the shaking table system are presented in Table 3.
The size of the model box was $2.80 \mathrm{~m}$ (length) $\times 1.40 \mathrm{~m}$ (width) $\times 1.80 \mathrm{~m}$ (height), and both sides were composed of U-shaped steel plates and plexiglass. A grid was used to divide the sidewall of the glass, which was convenient for observing the test phenomena and facilitated the sensor installation and removal. A polystyrene foam board was adhered to the back of the model box to prevent the reflection of seismic waves caused by rigid boundaries. To reduce the boundary effect of the model box, the bottom of the box was covered with $5 \mathrm{~cm}$ thick grit and crushed stone particles and was treated as a friction boundary to prevent the model and box from sliding against each other. A smaller polystyrene foam board adhered to the tunnel portal, which was treated as a sliding boundary to prevent the vibration of the box from affecting the tunnel structure. Additionally, the sidewall of the box was brushed with a layer of butter to eliminate its friction constraint. The width of the tunnel is $10 \mathrm{~m}$, and the clearance between the two tunnels is $7.47 \mathrm{~m}$ in the engineering prototype. Therefore, according to the geometric dimension ratio $C_{L}=1 / 50$, the tunnel width used in the model test is $20 \mathrm{~cm}$, the clear structure distance between the two tunnels is $15 \mathrm{~cm}$, and the tunnel length is $130 \mathrm{~cm}$. 


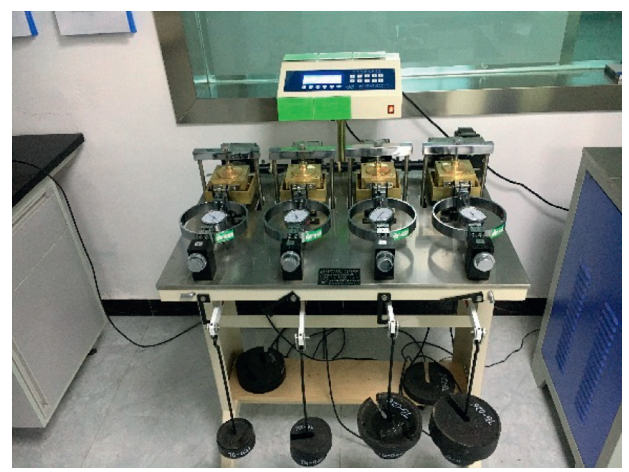

(a)

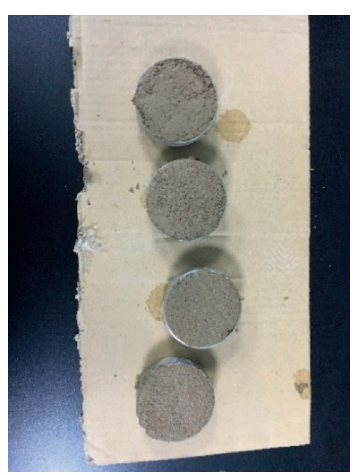

(b)

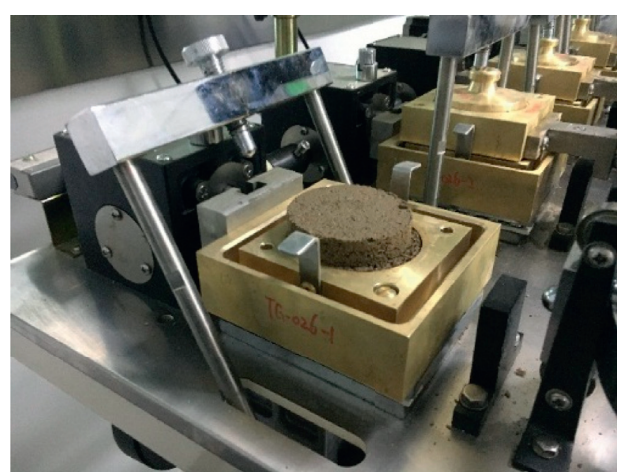

(c)

Figure 2: Test systems: (a) ZJ direct shear test system; (b) sample preparation; (c) the results of test.

TABLE 2: Physical and mechanical parameters of similar materials.

\begin{tabular}{|c|c|c|c|c|c|}
\hline Similar material & $\begin{array}{c}\text { Physical and mechanical } \\
\text { parameters }\end{array}$ & Elastic modulus, $E(\mathrm{GPa})$ & $\begin{array}{l}\text { Density, } \rho \\
\left(\mathrm{kg} / \mathrm{m}^{3}\right)\end{array}$ & Cohesion, $c(\mathrm{kPa})$ & $\begin{array}{c}\text { Internal friction angle, } \\
\phi\left(\left(^{\circ}\right)\right.\end{array}$ \\
\hline \multirow{2}{*}{ Surrounding rock } & Prototype & $1.3-6$ & $2000-2300$ & $200-700$ & $27-39$ \\
\hline & Model & 0.14 & 2270 & 10.47 & 34.80 \\
\hline \multirow{2}{*}{ Lining structure } & Prototype & 31.5 & 2300 & / & / \\
\hline & Model & 1.05 & 2180 & / & l \\
\hline
\end{tabular}

Table 3: Parameters of the shaking table system.

\begin{tabular}{lcc}
\hline Parameters & \multicolumn{2}{c}{$\begin{array}{c}\text { Technical } \\
\text { specification }\end{array}$} \\
\hline Table size $(\mathrm{m})$ & \multicolumn{2}{c}{$4 \times 6$} \\
Maximum bearing capacity $(\mathrm{t})$ & \multicolumn{2}{c}{25} \\
& \multicolumn{2}{c}{ Horizontal $(\mathrm{X})}$, \\
vertical $(\mathrm{Z})$, \\
Loading direction & horizontal-vertical \\
& \multicolumn{2}{c}{$(\mathrm{XZ})$} \\
& $\mathrm{X}$ & $0.1-70$ \\
Range of operating frequency $(\mathrm{Hz})$ & $\mathrm{Z}$ & $0.1-50$ \\
& $\mathrm{XZ}$ & $0.1-50$ \\
& $\mathrm{X}$ & 1.7 \\
Maximum acceleration $(\mathrm{g})$ & $\mathrm{Z}$ & 1.2 \\
& $\mathrm{XZ}$ & 1.2 \\
& $\mathrm{X}$ & \pm 250 \\
Maximum displacement $(\mathrm{mm})$ & $\mathrm{Z}$ & \pm 100 \\
& $\mathrm{XZ}$ & \pm 150 \\
\hline
\end{tabular}

To study the similarities and differences of the seismic responses of different cross-shaped three-dimensional tunnels under the same frequency and working conditions, the model was set up with the left and right sides (orthogonal- and oblique-type, respectively). This paper mainly focused on the orthogonal overlapped tunnel, and the similarities and differences of the oblique overlapped tunnel will be shown in other articles. The orthogonal overlapped tunnel model was laid out when the model box boundary processing was completed. Details are as follows: (1) The mixed surrounding rock material was filled in layers and compacted with tamping hammer. The density and moisture content of surrounding rock materials at different positions were selected to ensure the consistency of soil parameters from top to bottom. (2) The under-crossing tunnel was placed perpendicular to the left and right sidewalls of the model box after filling to a distance of $20 \mathrm{~cm}$ from the bottom of box. (3) Simultaneously, the corresponding accelerometers were embedded. (4) The upper-span tunnel was then placed parallel to the left and right sides of the model box after layering and filling $15 \mathrm{~cm}$. (5) Finally, the slope shape was built according to a slope ratio of the engineering prototype $(1: 2)$. The model layout is shown in Figure 3.

2.5. Accelerometer Arrangement and Measurement Points. TST120A500 accelerometers with a sensitivity of $500 \mathrm{mV} / \mathrm{g}$, a measurement range of $10 \mathrm{~g}$, and a frequency response range of $0.2-2500 \mathrm{~Hz}$ were used in the test. We mainly studied the dynamic response characteristics of the peak acceleration at the crown and invert of the upper-span and under-crossing tunnels in the orthogonal cross structure. The crown and invert of Strawberry ditch tunnel No. 2 (under-crossing tunnel) and the Pandaoling Highway tunnel (upper-span tunnel) were selected as the targets of the study. Accelerometers were arranged in the corresponding sections. To avoid the difference in the analysis results for the seismic response caused by the shape of the tunnel and consider both economy and timeliness, circular tunnels were used instead of hoof tunnels in the test [33]. The accelerometers were arranged symmetrically, as shown in Figure 4. Accelerometers of the upper-span tunnel were labeled as SA (crown: SA1-3, invert: SA4-6), and the under-crossing tunnel accelerometers were labeled as XA (crown: XA1-3, invert: XA4-6).

2.6. Design of Seismic Wave Loading. We considered the design of the dynamic response of the overlapped tunnel under various seismic intensities and various working 


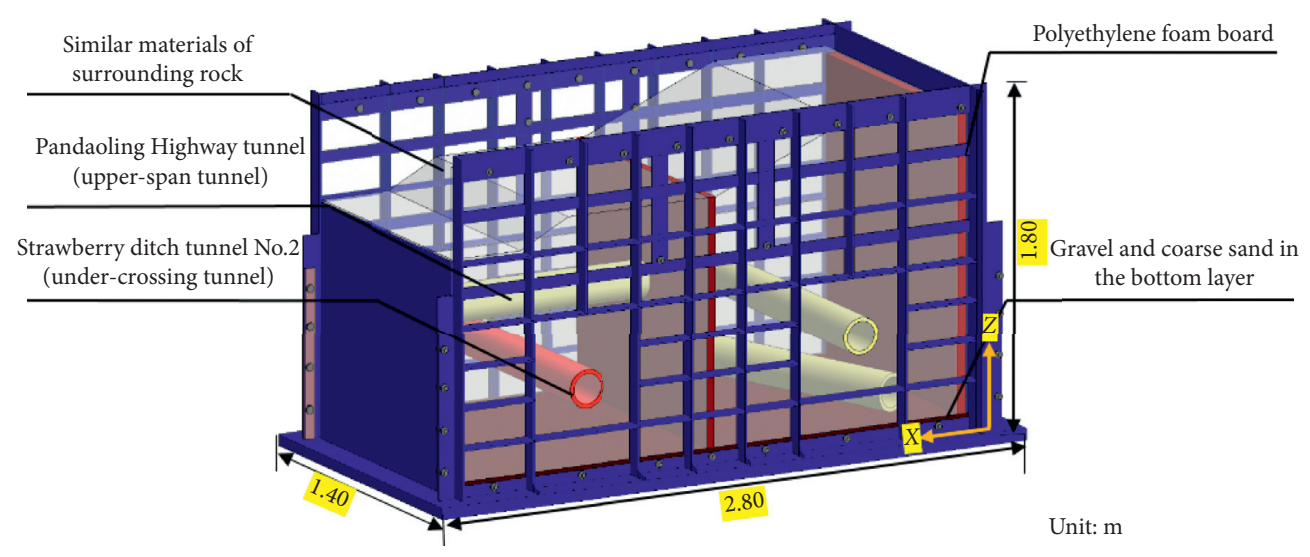

(a)

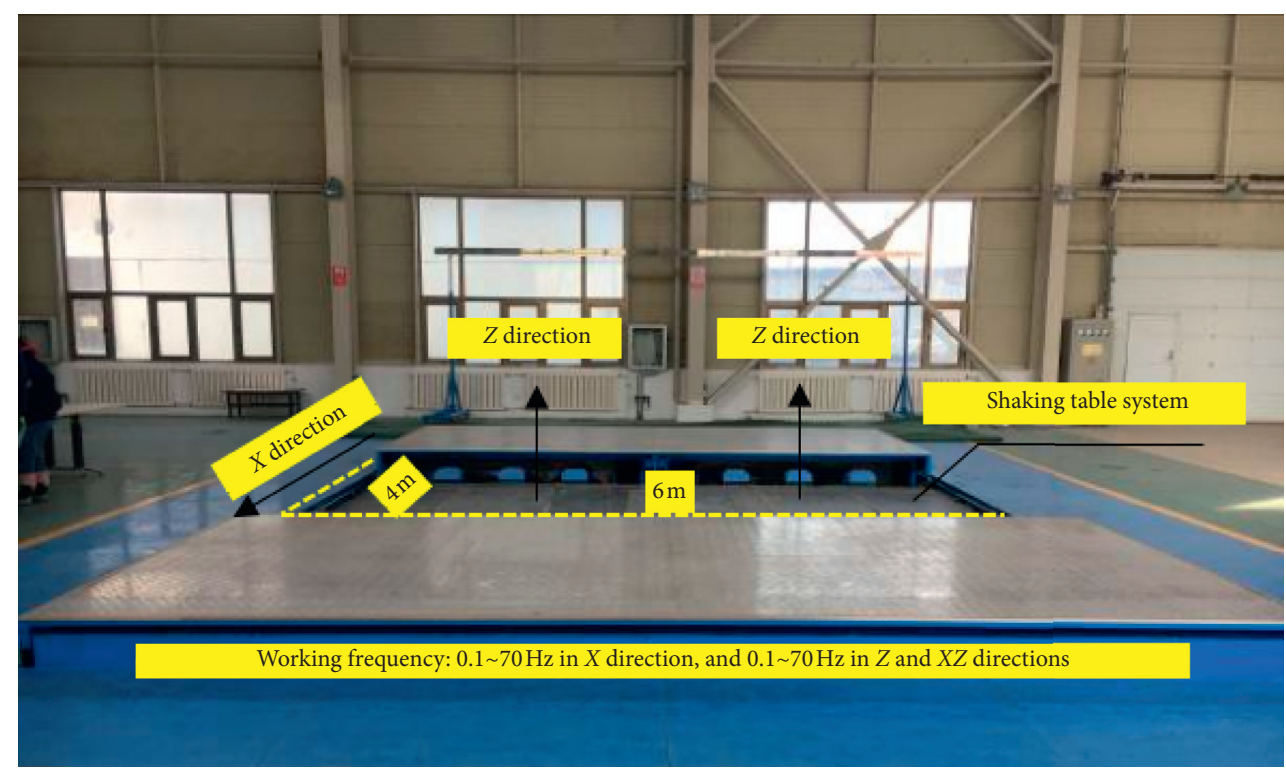

(b)

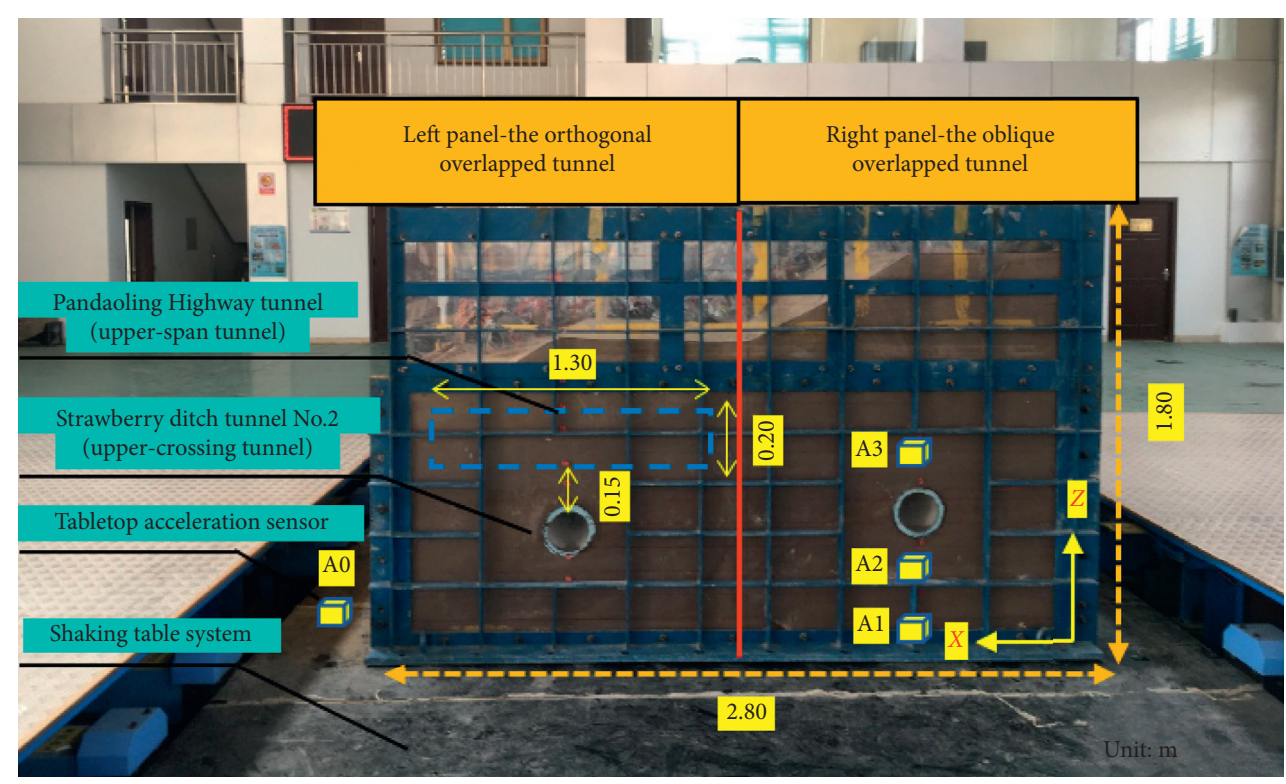

(c)

Figure 3: Layout of the test model: (a) sketch map; (b) shaking table system; (c) model map. 


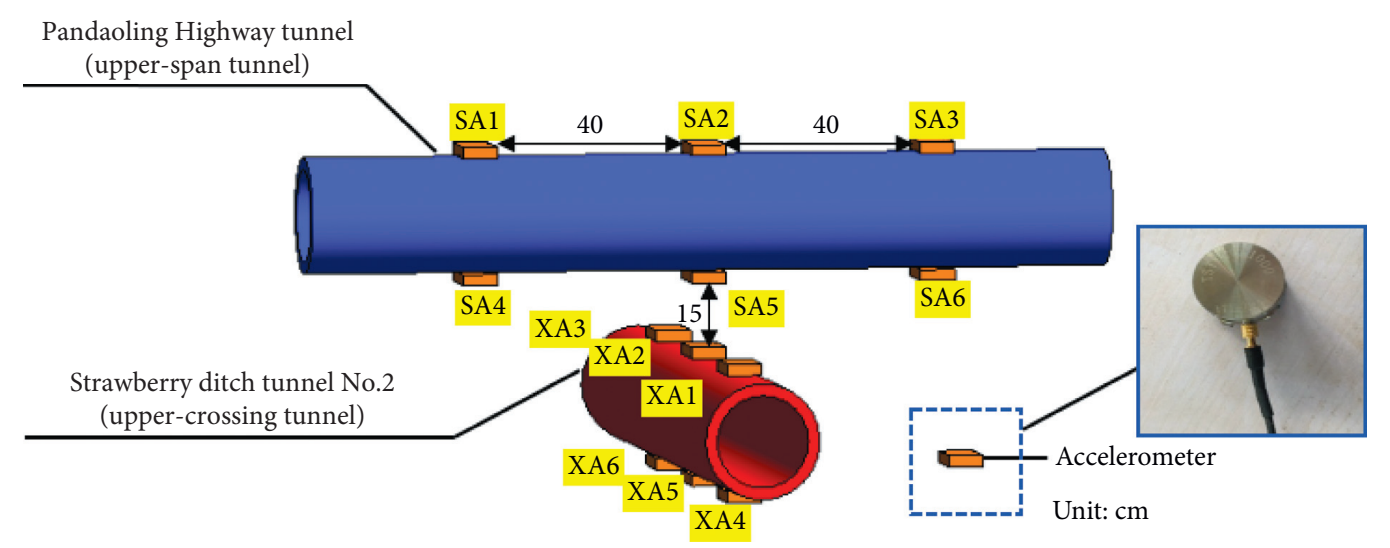

Figure 4: Arrangement of the accelerometers.

conditions in the shaking table test of this subject. During the loading process, sine waves, EL Centro in $X$ and $\mathrm{XZ}$ directions, and $\mathrm{WC}$ waves in $X$ and $\mathrm{XZ}$ directions were input, respectively, as shown in Figure 5. What we need to clarify is that, from the beginning of the test to the complete destruction of the model, the model has not been artificially restored to the initial state, and the overall model dynamically changes with the working conditions. However, owing to time and energy, we have currently only carried out a complete analysis of the $X$-directional WC wave with a relatively strong response after preliminary data processing and screening. The WC waves were recorded at Tangyu during the 2008 WC earthquake (Ms8.0) in Sichuan, China, whose original PGA and fault distances were $0.55 \mathrm{~g}$ and $10.1 \mathrm{~km}$, respectively $[34,35]$. The time history and Fourier spectrum of the WC wave are shown in Figure 6.

The test model was applied to 11 excitation cases, and the WC wave was inputted in the horizontal $(X)$ direction. The maximum input acceleration of the input seismic wave was $0.1 \mathrm{~g}, 0.15 \mathrm{~g}$ (seismic intensities of grade VII), $0.2 \mathrm{~g}, 0.3 \mathrm{~g}$ (grade VIII), and $0.4 \mathrm{~g}$ (grade IX), respectively, as shown in Table 4. It was set according to the principle of low seismic accelerations, followed by high seismic accelerations. Additionally, to test the dynamic characteristics of the system and study the damage of the system with the input seismic wave, a sine sweep (with maximum input acceleration of $0.05 \mathrm{~g}$ ) was inputted, while the amplitude of the input seismic wave was changed.

\section{Experiment Results}

3.1. Verification of the Boundary Condition. Combined with previous studies, Chen et al. [36] and Wu et al. [37] through calculation, analysis, and testing had shown that the flexible container can reproduce the prototype response well and had a lighter weight, which could meet the requirements of multidirectional vibration testing and weakened the boundary effect. Since the rigid model box was used in this test, two measures were taken to control the boundary condition design: (1) Control the plane size of the structure model. Generally, when the ratio of the foundation plane size to the structure plane size is greater than 5 , the error of the numerical calculation results caused by the lateral boundary

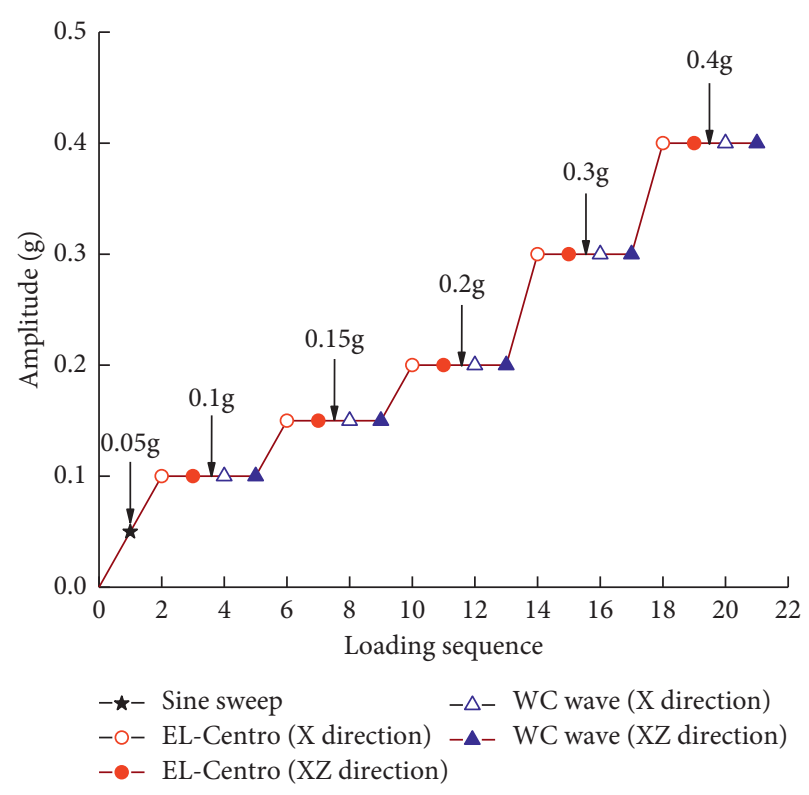

FIgURE 5: Loading sequence and amplitude.

is small and tends to be stable. The horizontal direction $(140 \mathrm{~cm})$ of the test is approximately 7 times the diameter of the structure tunnel $(20 \mathrm{~cm})$, which meets the requirements. (2) Use flexible materials on the side wall of the container to reduce the reflection effect of seismic waves at the boundary of the model box. Therefore, a layer of $50 \mathrm{~mm}$ thick highstrength polystyrene foam board was added to the inner walls of the horizontal vibration direction of the model box to reduce the reflection of the vibration wave, and there was a flexible foam partition in the center of the model box.

To verify the effect of this method on boundary effect, under the action of $0.1 \mathrm{~g}$ seismic wave, sine wave, EL Centro wave, and WC wave were successively inputted on the shaking table, and the seismic acceleration time history and Fourier spectra curves of measuring points A0 (table surface), A1, A2, and A3 (Figure 3) in the horizontal vibration direction of the model foundation surface were obtained. We selected the loading situation of the sine wave for analysis, as shown in Figure 7. 


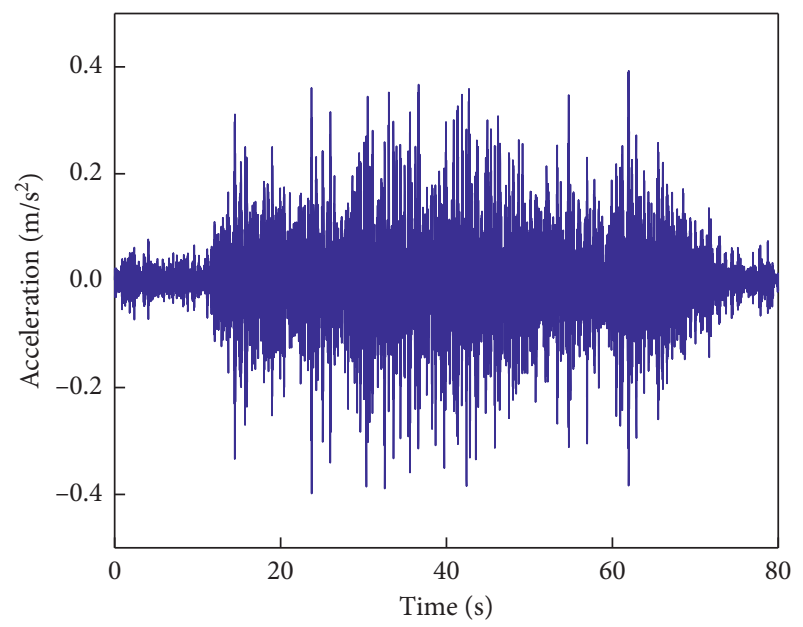

(a)

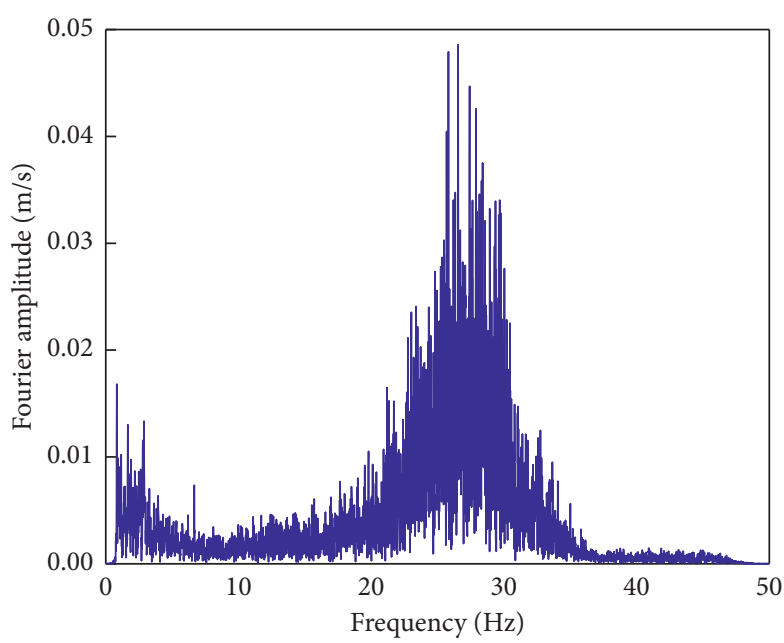

(b)

FIgURE 6: WC wave: (a) time history; (b) Fourier spectrum.

TABle 4: Seismic wave input cases.

\begin{tabular}{|c|c|c|c|c|}
\hline Number & Input sequence & Maximum input acceleration $(\mathrm{g})$ & Input direction & Seismic intensity \\
\hline \multirow{3}{*}{ Case 1} & Sine sweep & 0.05 & $X$ & - \\
\hline & WC wave & 0.1 & $X$ & VII \\
\hline & Sine sweep & 0.05 & $X$ & - \\
\hline \multirow{2}{*}{ Case 2} & WC wave & 0.15 & $X$ & VII \\
\hline & Sine sweep & 0.05 & $X$ & - \\
\hline \multirow{2}{*}{ Case 3} & WC wave & 0.2 & $X$ & VIII \\
\hline & Sine sweep & 0.05 & $X$ & - \\
\hline \multirow{2}{*}{ Case 4} & WC wave & 0.3 & $X$ & VIII \\
\hline & Sine sweep & 0.05 & $\mathrm{X}$ & - \\
\hline \multirow{2}{*}{ Case 5} & WC wave & 0.4 & $\mathrm{X}$ & IX \\
\hline & Sine sweep & 0.05 & $\mathrm{X}$ & - \\
\hline
\end{tabular}

We can obtain that the acceleration sensors at different positions of the model soil have quite similar curves under the same seismic wave, and the time for each measuring point to reach peak acceleration is basically the same. At the same time, comparing the acceleration response Fourier spectrum of each measuring point, the result shows that the Fourier spectrum composition of different measuring points under the same seismic wave action is close to the spectrum value. From Figure 7, the sampling time interval is $0.001 \mathrm{~s}$, the peak acceleration is $1.2-1.8 \mathrm{~m} / \mathrm{s}^{2}$, the main vibration time is about 23 to $78 \mathrm{~s}$, and the total time is $55 \mathrm{~s}$. The excellent frequency is about $1.0 \mathrm{~Hz}$, and the maximum amplitude is about $0.05-0.07$. It shows that the polystyrene foam board installed on the wall of the model box perpendicular to the vibration direction can eliminate the reflection and scattering of waves on the boundary and has a better effect on reducing the boundary effect.

Moreover, we introduced the concepts of relative error and boundary effect index to quantitatively describe the degree of dispersion between the accelerations of each measuring point and verify the processing effect of the model box boundary [38]. The calculation method of relative error and boundary effect index is as follows:

$$
\begin{aligned}
& \delta_{x, y}=\left|\frac{X-Y}{X}\right| \times 100 \%, \\
& S_{x, y}=\sqrt{\frac{\sum\left(x_{i}-y_{i}\right)^{2}}{n-1}}, \\
& \mu_{x, y}=\frac{S_{x, y}}{X} \times 100 \%,
\end{aligned}
$$

where $\delta_{x, y}$ is relative error; $S_{x, y}$ is standard deviation; $\mu_{x, y}$ is boundary effect coefficient; $x_{i}$ is the acceleration sample value at a position far away from the wall of the model box; here the acceleration of $\mathrm{A} 3$ is the reference value; $X$ is the peak acceleration of the measuring point; $y_{i}$ is the acceleration sample value at other positions; $Y$ is the peak acceleration of the corresponding measuring point.

The acceleration of A3 is selected as the reference value to calculate the various indicators of the boundary effect. Table 5 shows the specific values of the boundary effect verification index when sine wave, EL Centro wave, and WC wave are inputted under $0.1 \mathrm{~g}$ of maximum input acceleration. 

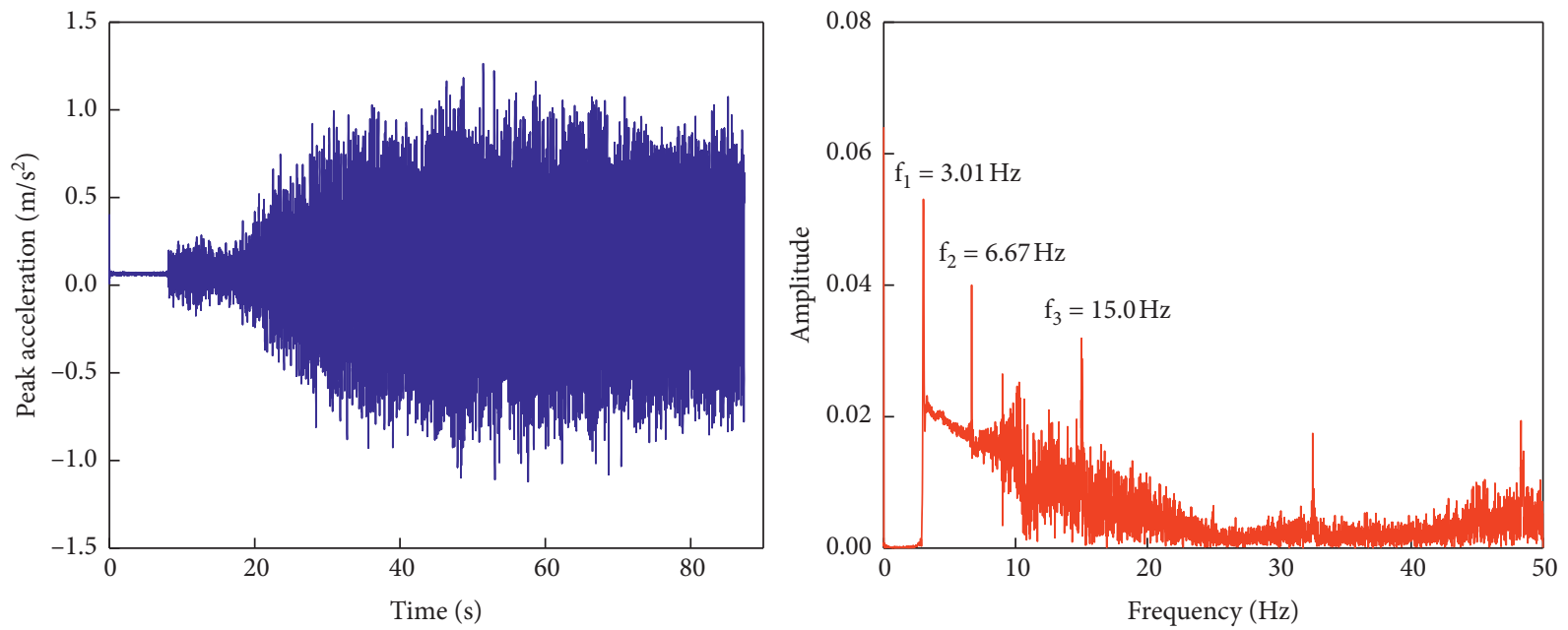

(a)
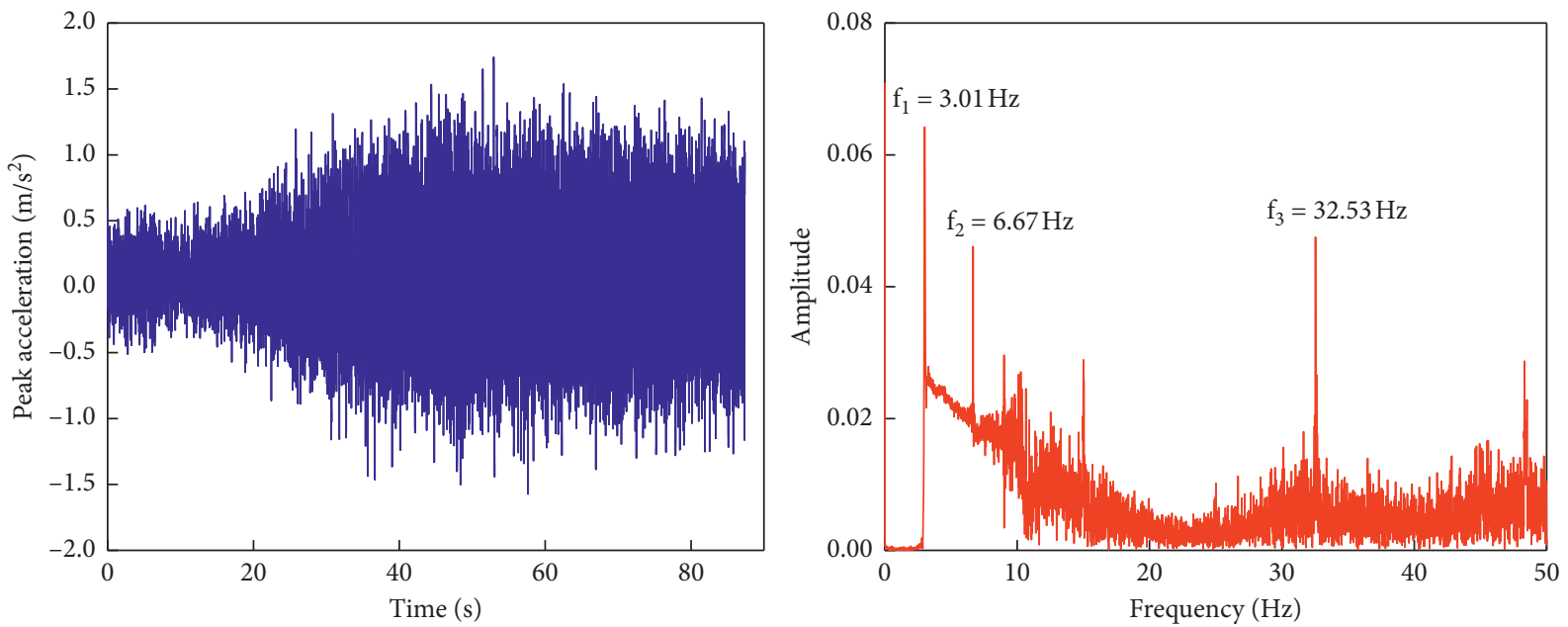

(b)
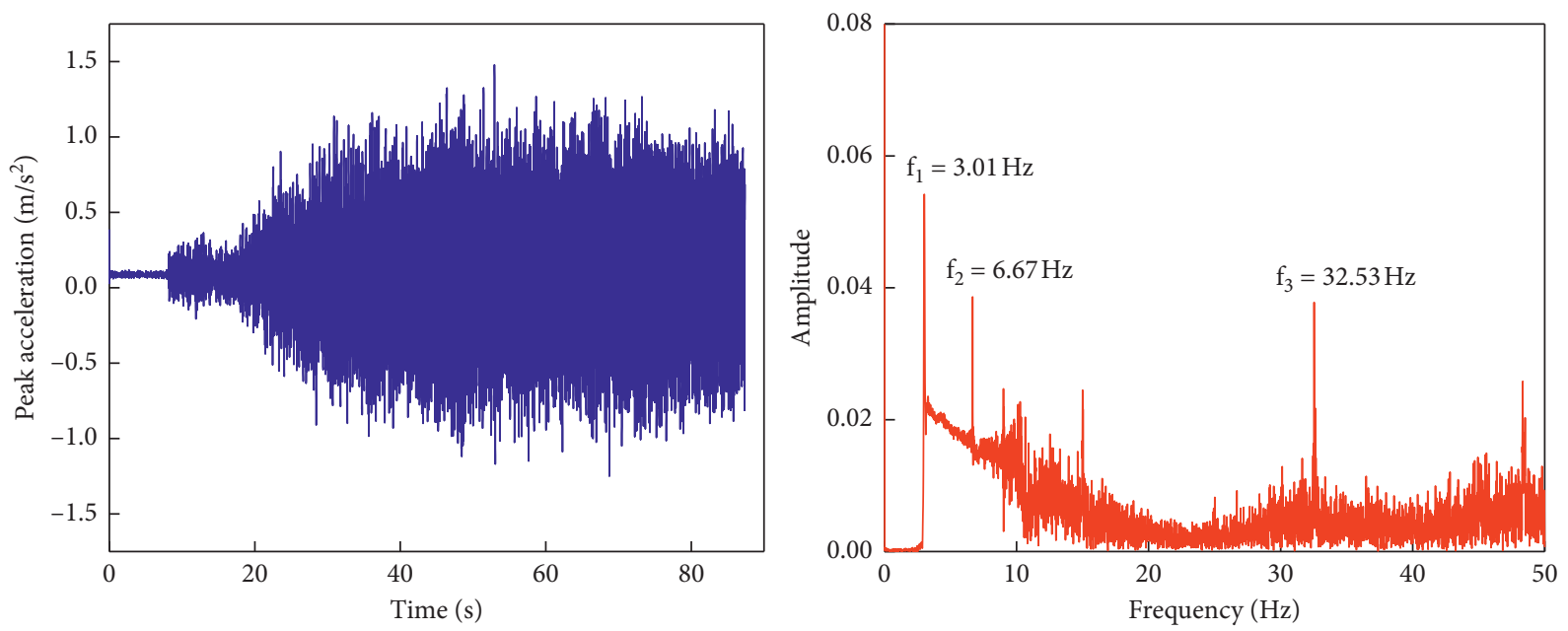

(c)

FIgURE 7: Continued. 

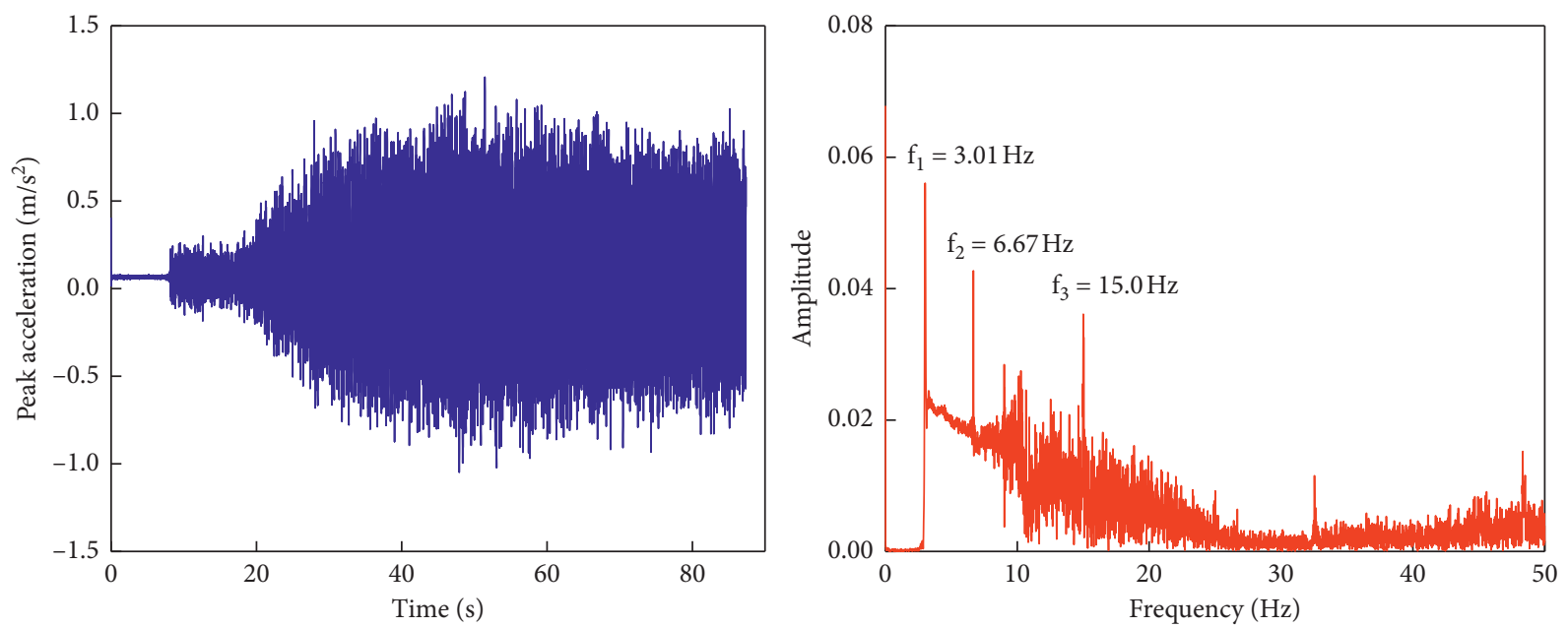

(d)

FIGURE 7: Acceleration time domain and frequency spectrum curves at different positions when sine wave of $0.1 \mathrm{~g}$ is inputted in $X$ direction. (a) A0. (b) A1. (c) A2. (d) A3.

TABLE 5: Verification indicators of boundary effect.

\begin{tabular}{lcccccccccc}
\hline \multirow{2}{*}{ Input seismic wave } & \multicolumn{3}{c}{ Peak acceleration $\left(\mathrm{m} / \mathrm{s}^{2}\right)$} & \multicolumn{3}{c}{ Relative error (\%) } & \multicolumn{3}{c}{$\begin{array}{c}\text { Boundary effect coefficient } \\
(\%)\end{array}$} \\
& $\mathrm{A} 0$ & $\mathrm{~A} 1$ & $\mathrm{~A} 2$ & $\mathrm{~A} 3$ & $\delta_{0,3}$ & $\delta_{1,3}$ & $\delta_{2,3}$ & $\mu_{0,3}$ & $\mu_{1,3}$ & $\mu_{2,3}$ \\
\hline Sine wave & 1.26 & 1.63 & 1.32 & 1.47 & 14.28 & 10.88 & 10.20 & 9.71 & 7.40 \\
EL Centro wave & 0.87 & 1.04 & 0.89 & 0.96 & 9.37 & 8.33 & 7.29 & 9.76 & 8.67 & 7.59 \\
WC wave & 0.98 & 0.87 & 0.89 & 0.92 & 6.52 & 5.43 & 3.26 & 7.08 & 5.90 & 3.54 \\
\hline
\end{tabular}

It can be seen from the verification index of boundary effect coefficient that, under the action of arbitrary seismic wave, the farther away position from the base reference value, the larger the boundary effect coefficient, showing the overall rule of $\mu_{0,3}>\mu_{1,3}>\mu_{2,3}$, and the boundary effect coefficients are all less than $10 \%$. Some studies [39] showed that when the boundary coefficient was less than $10 \%$, the scattering and reflection of seismic wave at the boundary could be eliminated. The model box boundary perpendicular to the vibration direction has a good treatment effect in this test. Therefore, we deem that the boundary effect on both ends of the tunnel is effectively controlled within the acceptable error range.

Furthermore, sine sweep was generally used to test the dynamic response of the system to obtain the influence of the natural frequency and damping ratio of the slope on the dynamic parameters of the test model during the test. Therefore, we performed fast Fourier transform (FFT) on the acceleration of each measuring point under the random sweep of a low amplitude $(0.1 \mathrm{~g})$ sine wave and analyzed the corresponding spectrogram to obtain the natural frequency of the model. From the FFT spectrum diagram in Figure 7, it can be seen that the spectrum change law of each measurement point basically shows the same law. The first-order natural frequency of the model is $3.01 \mathrm{~Hz}$, which is the fundamental frequency of the model. At this frequency, its amplitude also reaches its maximum, which indicates that the fundamental frequency coincides with the main frequency; that is, natural frequency of the model is $3.01 \mathrm{~Hz}$. The second-order natural frequency of the model is $6.67 \mathrm{~Hz}$, while the third-order natural frequencies of each measuring point behave slightly differently, being $15.0 \mathrm{~Hz}$ and $32.53 \mathrm{~Hz}$, respectively. However, second- and third-order frequencies are not natural frequencies, which may be the excitation frequency or its multiples.

\subsection{Acceleration Response of Upper-Span Tunnel. From the} perspective of seismic wave energy transfer, the higher the strength and rigidity of the surrounding rock and structure, the more conducive to energy transfer, and the acceleration amplification effect is more obvious along the slope body in all directions and the empty surface. Therefore, the acceleration response is closely related to the local damage mode of surrounding rock and structures.

Take Cases $1-5$, which were inputted in $X$ direction, as examples. The peak accelerations for different measured locations in the crown and invert of the upper-span tunnel are shown in Figures 8(a) and 8(b), respectively. In general, the response acceleration increased with the maximum input acceleration of the input seismic wave.

When the model was excited by the WC waves with different intensities (i.e., $0.1 \mathrm{~g}, 0.15 \mathrm{~g}$, and $0.2 \mathrm{~g}$ ), the seismic response at SA2 of the center of the crown was smaller than that at the two sides of SA1 and SA3, where the peak acceleration was distributed in a "V" shape. The peak acceleration distribution at SA3 was the largest. However, at grades VIII-IX $(0.3 \mathrm{~g}-0.4 \mathrm{~g})$, the peak acceleration 


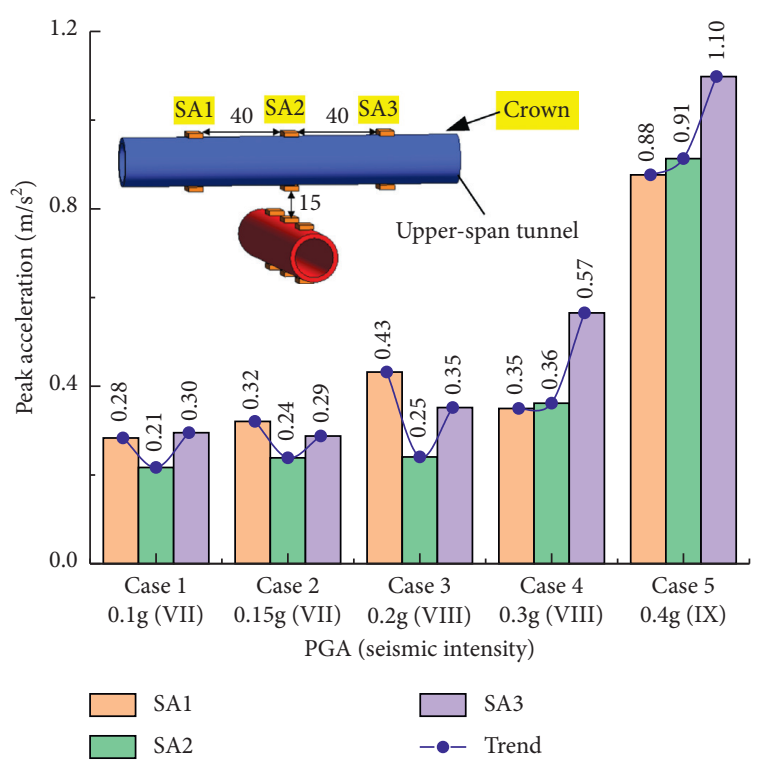

(a)

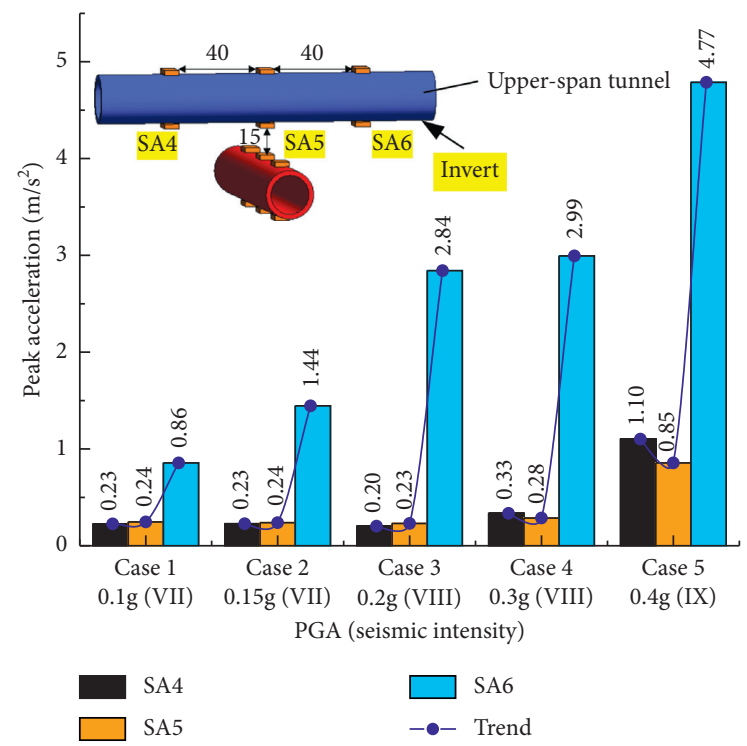

(b)

Figure 8: Peak acceleration of the upper-span tunnel: (a) crown; (b) invert.

distribution of the crown exhibited slight changes, where the peak acceleration increased nonlinearly with respect to the seismic intensity. The reason for this phenomenon is that the seismic wave is horizontally loaded and SA3 is closer to the source and receives the seismic wave earlier and stronger, so its dynamic response is larger than that of SA2. Additionally, SA1 of the affected section upper-span tunnel is closer to the empty surface of the slope, and the soil cover thickness is relatively thin, which will cause obvious deformation under the action of larger seismic waves. For the invert of the upper-span tunnel, the peak acceleration exhibited a nonlinear increase when the range of the input seismic wave was $0.1 \mathrm{~g}-0.2 \mathrm{~g}$ (Cases 1-3). From the perspective of seismic wave energy transfer, as the seismic wave transmits horizontally, part of energy is absorbed by the surrounding rock and tunnel structure and energy transfer is weakened sharply. The acceleration response showed an approximate parabolic linear decline along the horizontal path: SA6 $\longrightarrow$ SA5 $\longrightarrow$ SA 4 under $0.1 \mathrm{~g}-0.2 \mathrm{~g}$. With the increase of the maximum input acceleration, the acceleration response has an obvious amplifying effect on the free surface in the horizontal direction; that is, the acceleration response of SA4 increases, showing an obvious "surface trending effect."

Moreover, we have also analyzed the deformation and failure of the model, and the experimental results are basically consistent with our inferences. During the loading process where the maximum input acceleration is $0.1 \mathrm{~g}-0.15 \mathrm{~g}$ (seismic intensity is VII), slight cracks appeared around the tunnel. With continued loading, the longitudinal cracks and transverse cracks on both sides of the upper-span tunnel crown continued to expand and developed, with longitudinal cracks reaching $8 \mathrm{~mm}$ and transverse cracks reaching $5 \mathrm{~mm}$, as shown in Figures 9(a) and 9(b). At this time, the model as a whole has basically begun to be in the deformation stage.
We define the amplification factor as the ratio between the peak acceleration value of the measured acceleration and the peak acceleration value of the input. The amplification factors of different measurement points in the upper-span tunnel for various cases are presented in Figure 10. When the seismic intensity was relatively low $(0.1 \mathrm{~g}-0.15 \mathrm{~g})$, that is, under the excitation of small amplitude seismic waves, the surrounding rock of the slope was in the elastic stage, and the acceleration amplification factor of each measured point exhibited the characteristics of linearity and stationarity increase. When the peak value of input seismic wave is $0.15 \mathrm{~g}-0.2 \mathrm{~g}$, the acceleration amplification factor of each measured point increased, which can be regarded as the plastic state of the model. However, after the input of $0.2 \mathrm{~g}$ seismic wave, the relationship curve between the acceleration amplification factor and the peak value of the input wave presented a "sawtooth" distribution state. The reason is analyzed that, after the action of the $0.2 \mathrm{~g}$ seismic wave, the surrounding rock of the slope has been locally damaged, and the change of the slope geometry and force status has an impact on the propagation law of acceleration. When the peak value of input wave was $0.2 \mathrm{~g}-0.3 \mathrm{~g}$, the acceleration amplification factor of each measured point decreased, which indicated that the dynamic shear modulus of soil decreased and the damping ratio increased in this process. It can be considered that the model is in a state of plastic enhancement at this time. When the amplitude of input seismic wave was $0.3 \mathrm{~g}-0.4 \mathrm{~g}$, the acceleration amplification factor of changed suddenly and the seismic response increased abnormally; we were convinced that as the cumulative damage of the tunnel surrounding rock inside the slope increased, cracks gradually increased (consumption of seismic energy), and the filtering effect became more and more intensified. In plastic enhancement stage, the amplitude of the dynamic stress intensity factor of the tunnel 


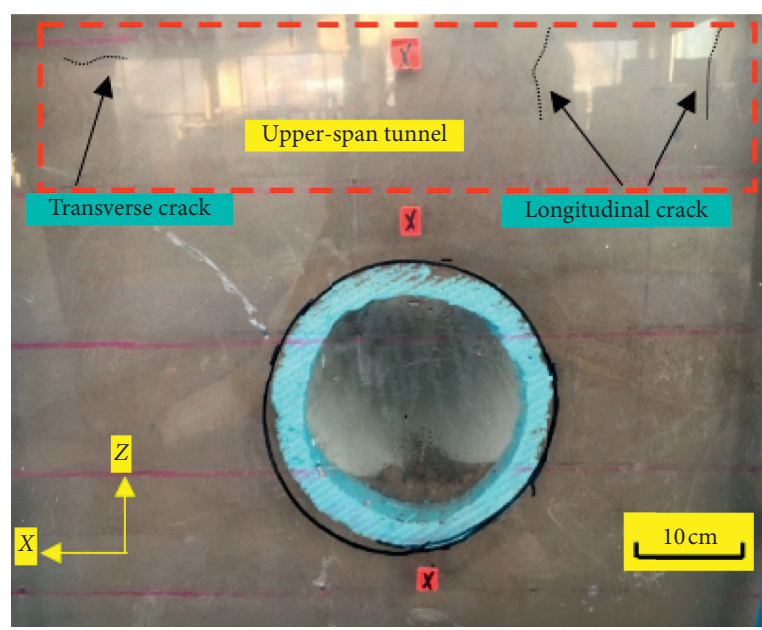

(a)

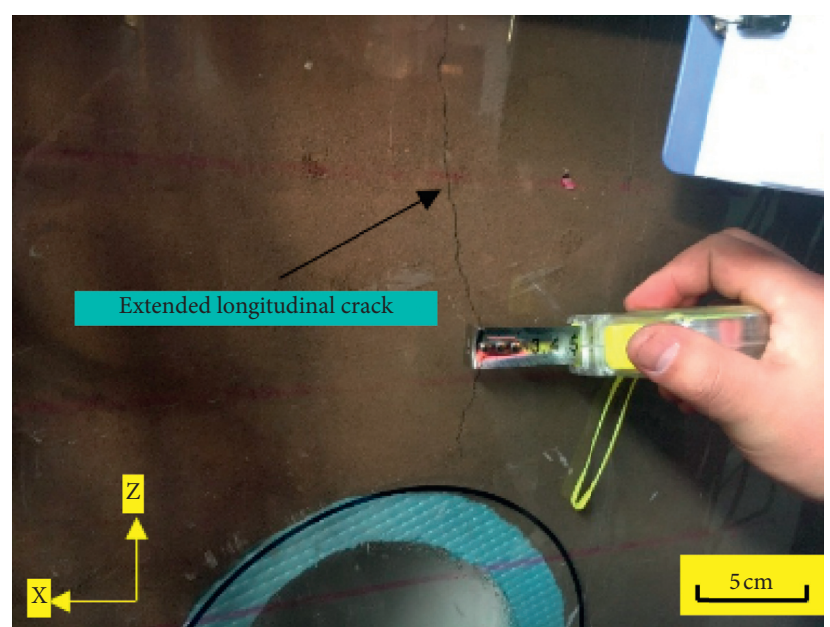

(b)

Figure 9: Model deformation and failure: (a) main view; (b) rear view.

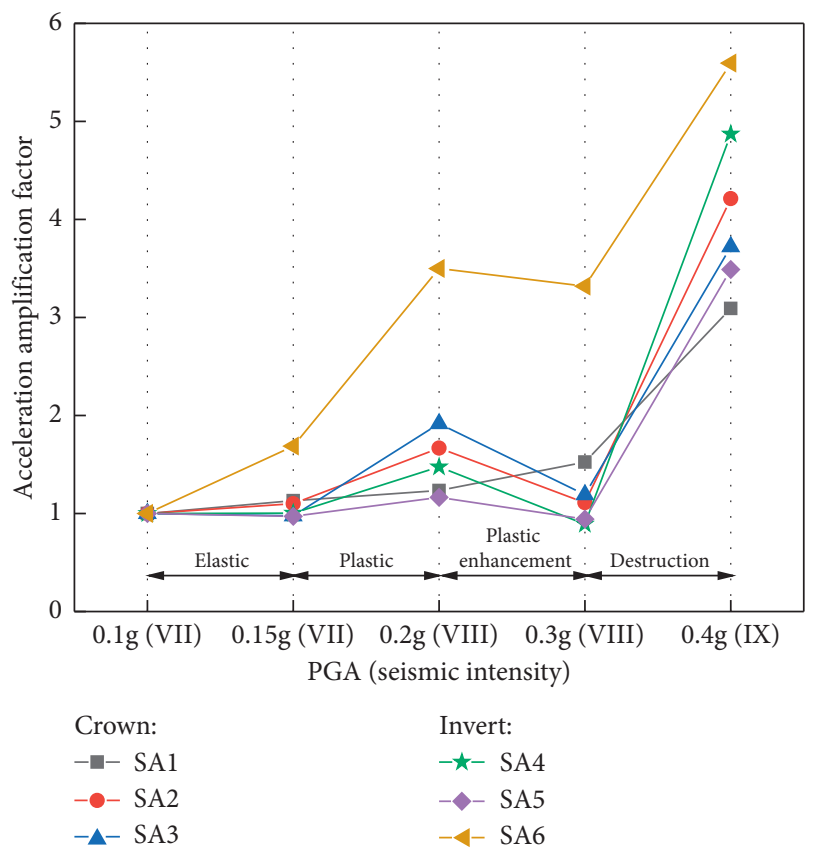

FIGURE 10: Acceleration amplification factor of the upper-span tunnel.

surrounding rock increased to its fracture toughness, resulting in a sharp decrease in the number of microscopic cracks and a significant increase in damage rate, and accelerated expansion into macroscopic cracks. Furthermore, the integrity of the surrounding rock was seriously damaged and its bearing capacity was significantly weakened (critical instability stage) and further penetration of macro cracks would promote the failure of the model.

Additionally, the growth of the seismic dynamic response at the crown of the cross section was significantly faster than that on both sides of the affected section when the seismic intensity was VIII-IX (0.3-0.4 g), indicating that the cross section within the intensity range was a weak area. The damage of the invert evolved from the right side to the cross section and then to the left side for grades VII-VIII (0.1-0.2 g) but developed from the affected section on both sides to the cross section for grades VIII-IX (0.3-0.4 g). Although this situation is relatively rare in real world, we are convinced that the study results can provide some references for similar situations as a qualitative study of the seismic response of overlapping tunnels.

3.3. Acceleration Response of Under-Crossing Tunnel. The distributions of the peak values of the acceleration from the two directions at the under-crossing tunnel are compared in Figure 11. The response acceleration of the under-crossing tunnel increased with the maximum input acceleration of the input seismic wave.

For the crown of the under-crossing tunnel, the peak acceleration exhibited a parabolic shape when the maximum input acceleration of $0.1 \mathrm{~g}-0.3 \mathrm{~g}$ was inputted, and the response of XA1 in the affected section was the strongest, followed by that of XA2. However, the distribution was different from the previous cases when a maximum input acceleration of $0.4 \mathrm{~g}$ was inputted, and the peak acceleration of XA3 in the affected section was the highest. Furthermore, for the invert of the under-crossing tunnel, the seismic response of XA5 in the cross section of the invert was smaller than that of the two measurement points on both sides of the affected section, with a "V" distribution. Thus, the peak acceleration of XA5 was lower than those of XA4 and XA6. Owing to the existence of the upper-span tunnel and the spatial effect, the seismic response of the invert of the undercrossing tunnel at the cross section of the under-crossing tunnel was significantly weaker than that of the crown.

Similarly, the amplification factors of different measurement points in the under-crossing tunnel for various cases are presented in Figure 12. We found that the deformation mechanism of the under-crossing tunnel is similar to that of the upper-span tunnel; namely, the model exhibited obvious deformation stages during the seismic wave loading process, which can be divided into elastic, 


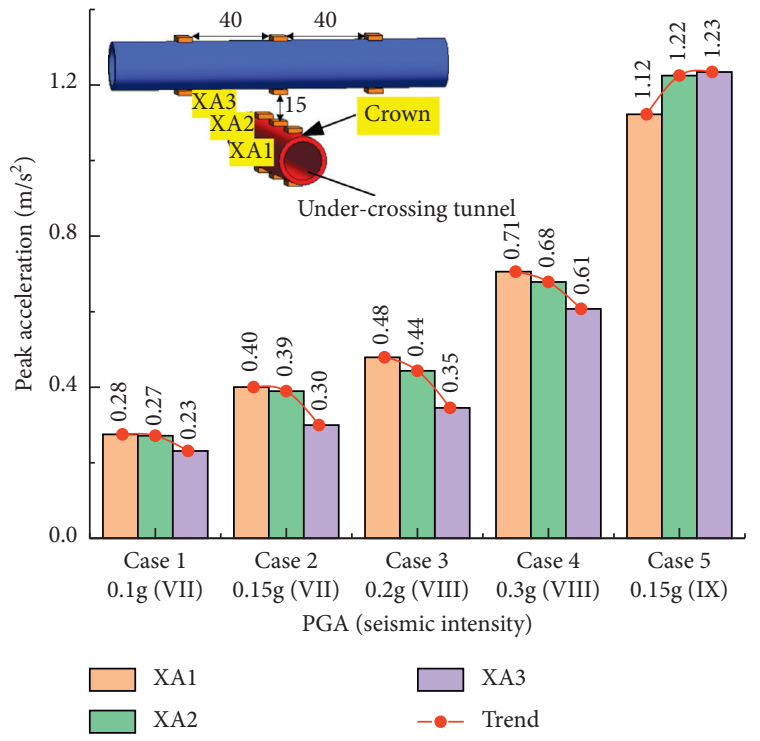

(a)

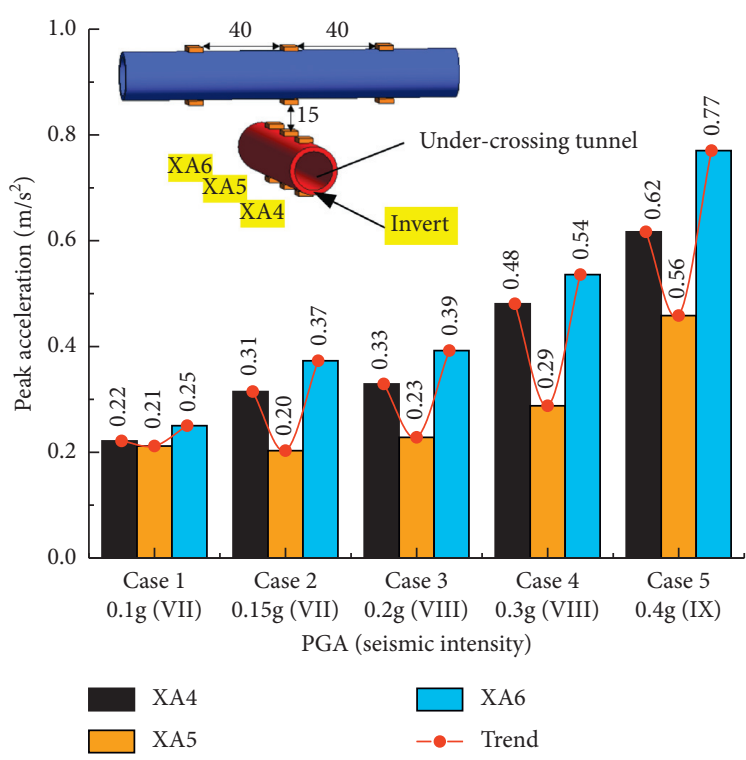

(b)

FIgURE 11: Peak acceleration of the under-crossing tunnel: (a) crown; (d) invert.

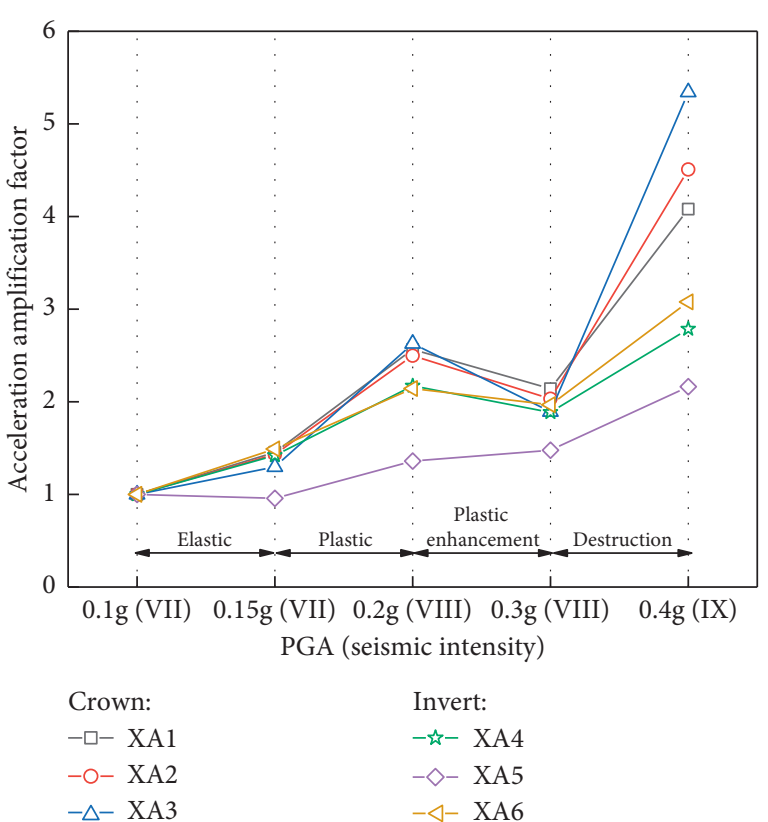

Figure 12: Acceleration amplification factor of the under-crossing tunnel.

plastic, plastic enhancement, and failure stage. As shown in Figure 12, the acceleration amplification factor increased steadily and slowly at low seismic accelerations, but, with a continuous increase in the seismic intensity, the amplification factor increased sharply. Since the seismic ground motion was inputted in the horizontal direction ( $X$ direction), its longitudinal stiffness was relatively large for the homogeneous ring model. Therefore, the acceleration response of each point in the tunnel showed basically the same law under the seismic uniform ground motion when maximum input acceleration was $0.1-0.3 \mathrm{~g}$. However, the measurement points showed obvious differences when the seismic intensity was large (maximum input acceleration is $0.4 \mathrm{~g}$ ); that is, the acceleration amplification factors of the crown decreased in the following order: XA3 $>$ XA2 $>$ XA1. After the model was subjected to multiple earthquakes, the vertical cracks around the tunnel surrounding rock and the secondary joints in the slope gradually expanded and penetrated the layer, resulting in increased cumulative damage to the tunnel surrounding rock. We inferred that the reason for this situation may be that the model had begun to deform and fail, which we could see from the sudden change in the acceleration magnification factor. The crown response of the under-crossing tunnel was more sensitive when the seismic intensity was higher. In addition, the acceleration amplification factors on the cross section were essentially coincident with the two sides of the affected section at the invert of the under-crossing tunnel. Therefore, the earthquake damage of the invert of the under-crossing tunnel developed from the affected section on both sides to the cross section under the horizontal seismic input.

Through analyzing the dynamic response characteristics of the model, it is helpful to further explore the working state of the structure and the development mode of seismic damage of the surrounding rock. We found that the model presented significant deformation stages during the seismic wave loading process, and the seismic response of the invert of the upper-span tunnel and the crown of the undercrossing tunnel for overlapped tunnel was relatively strong. Therefore, we can make a macro grasp of the safety performance of the tunnel engineering and avoid the occurrence of engineering accidents such as the paralysis of the entire line due to the destruction of the tunnel by improving the corresponding antiseismic level of the structure in advance. 


\section{Discussion}

4.1. Analysis of Continuous Wavelet Transform (CWT). Through the analysis of the frequency spectrum, we can obtain the relevant information of excellent frequency and frequency band, which can more clearly understand the frequency and energy distribution characteristics of the seismic wave transmission process. In engineering applications, the seismic waves in the main frequency bands should also be avoided to resonate with the structure, providing relevant theoretical references for engineering practice. Therefore, to study the seismic response and spectral characteristics of the overlapped tunnel in more detail, we introduced wavelet transform (WT), a mathematical tool that can also characterize the local characteristics of seismic waves in the time-frequency domain. The WT allows multiresolution analysis and can be used to reveal the local characteristics of the signal in both the time and frequency domains $[40,41]$. The WT is a mathematical function with zero mean that can be localized in the time and frequency domains [42]. By using a wavelet basis function to translate and scale the signal along with the time index, we can focus on any position and obtain all the details of the signal. The specific equations of the WT are as follows:

$$
\begin{aligned}
F(a, b) & =\int_{-\infty}^{+\infty} f(t) \psi *(t ; a, b) d t, \\
\psi(t ; a, b) & =\frac{1}{\sqrt{a}} \psi\left(\frac{t-b}{a}\right),
\end{aligned}
$$

where $f(t)$ represents the time-domain signal, $\psi(t ; a, b)$ represents the wavelet basis function, $*$ represents the complex conjugate, $t$ represents time, $a$ is the translation factor, and $b$ is the expansion factor.

The CWT allows multiscale analysis of the signal through the translation and expansion of the wavelet basis function, which is suitable for extracting features of the signal $[43,44]$. The frequency information of the signal at any scale and at any time can be obtained through the CWT. In this study, the Morlet wavelet, which has the resolution in the timefrequency joint domain as well as high-amplitude aggregation [45], was used. The Morlet wavelet function and wavelet waveform (Figure 13) by [46] are expressed as follows:

$$
\psi(t)=\sqrt[4]{\frac{1}{\pi}} \cos \left(2 \pi f_{0} t\right) e^{-\left(t^{2} / 2\right)},
$$

where $f_{0}=0.8125$ represents the center frequency of the wavelet basis function and $2 \pi f_{0}=5$ represents the wavenumber.

Owing to the existence of the cross section, the tunnel structure may be affected to some extent; thus, the dynamic response and spectral characteristics of the cross section of the orthogonal overlapped tunnel are discussed here. The Morlet wavelet was used to transform the invert of the central section of the upper-span tunnel (SA5) and the crown of the under-crossing tunnel (XA2), and its

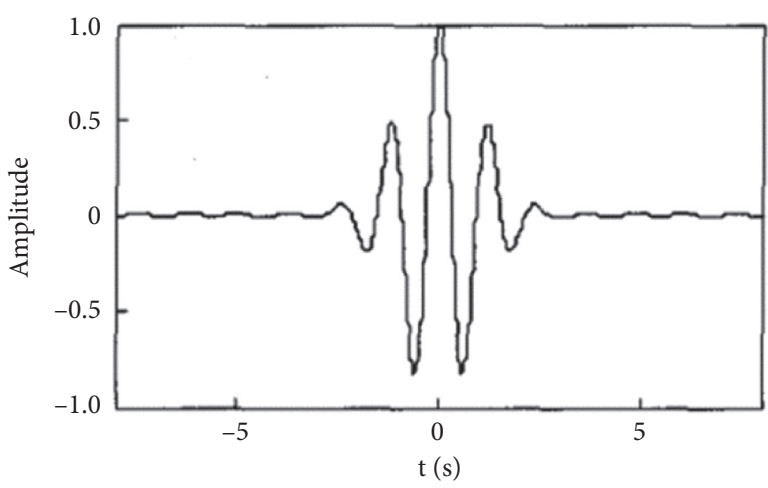

Figure 13: Waveform of the Morlet wavelet.

spectrum was analyzed. Analysis of the peak acceleration for various loading cases revealed that the seismic dynamic response changed obviously when it reached $0.2 \mathrm{~g}-0.3 \mathrm{~g}$ when loading $X$ direction of the WC wave. Therefore, the input with a maximum input acceleration of $0.2 \mathrm{~g}$ (grade VIII) was selected as the demarcation point for analysis.

Furthermore, the transform scale is an important parameter for the Morlet wavelet [47]. To thoroughly consider the spectrum changes at different scales, the proposed scales of change in this study were $1,2,5,10,20,50,100,500$, and 1000 , respectively.

4.2. CWT of the Invert (SA5) of Upper-Span Tunnel. Taking Case 8 (maximum input acceleration is $0.2 \mathrm{~g}$ ) as an example, the typical time history and Fourier spectrum of the invert of the upper-span tunnel transformed by SA5 are presented in Figure 14. From Figure 14, the acceleration and frequency characteristics of the original signal at the invert of the upper-span tunnel at different scales and positions can be obtained. For the acceleration dynamic response of the SA5 measurement point, the response of the transformation of small scales was relatively large in the $4-50 \mathrm{~Hz}$ range of the original signal. The acceleration response was relatively strong within 40-60 s after the seismic wave was inputted. Additionally, when the scale value of the transform was small, the time domain of the Morlet wavelet transform was relatively narrow, and the frequency domain was relatively wide. When the scale value of the transform was large, the opposite was observed: the time domain was relatively wide, and the frequency domain was narrow $[48,49]$.

To elucidate the relationship between the acceleration peak response and the frequency, the CWT at the transformation of small scales $(1,2,5,10$, and 50) was analyzed. We defined $R_{a i}$ and $R_{f i}$ (Table 6 ) as the peak acceleration and dominant-frequency ratios, respectively, of the transformation-scale signal to the original signal. That is, the closer the parameters are to 1 , it indicates that this frequency band or peak acceleration is the dominant parameter of the original signal. They are expressed as follows: 


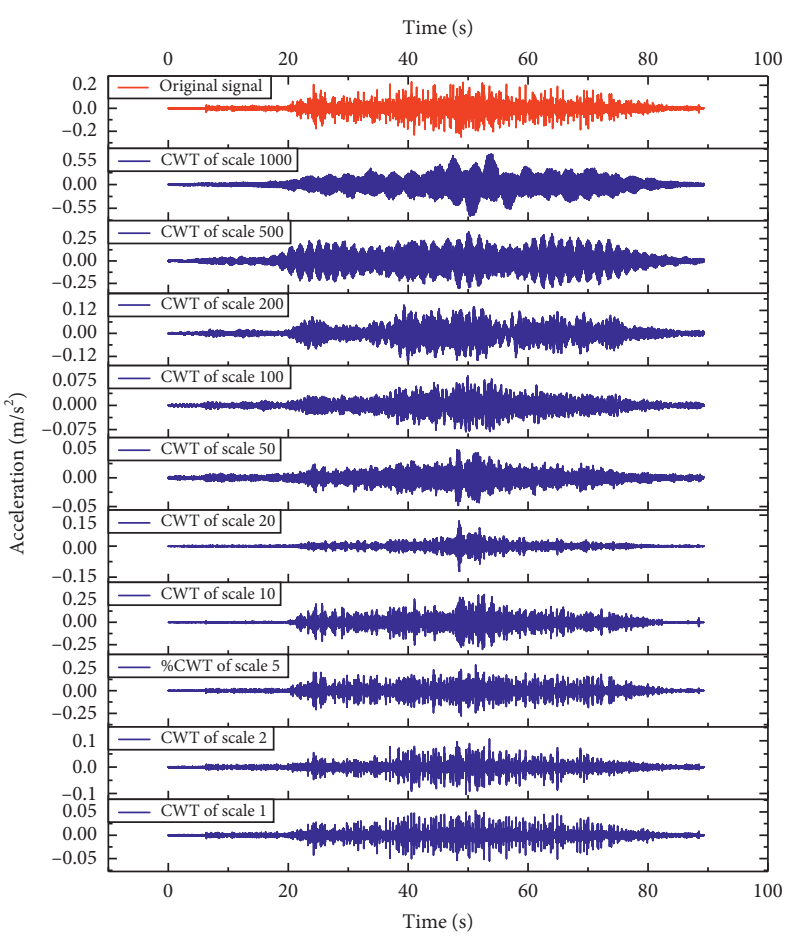

(a)

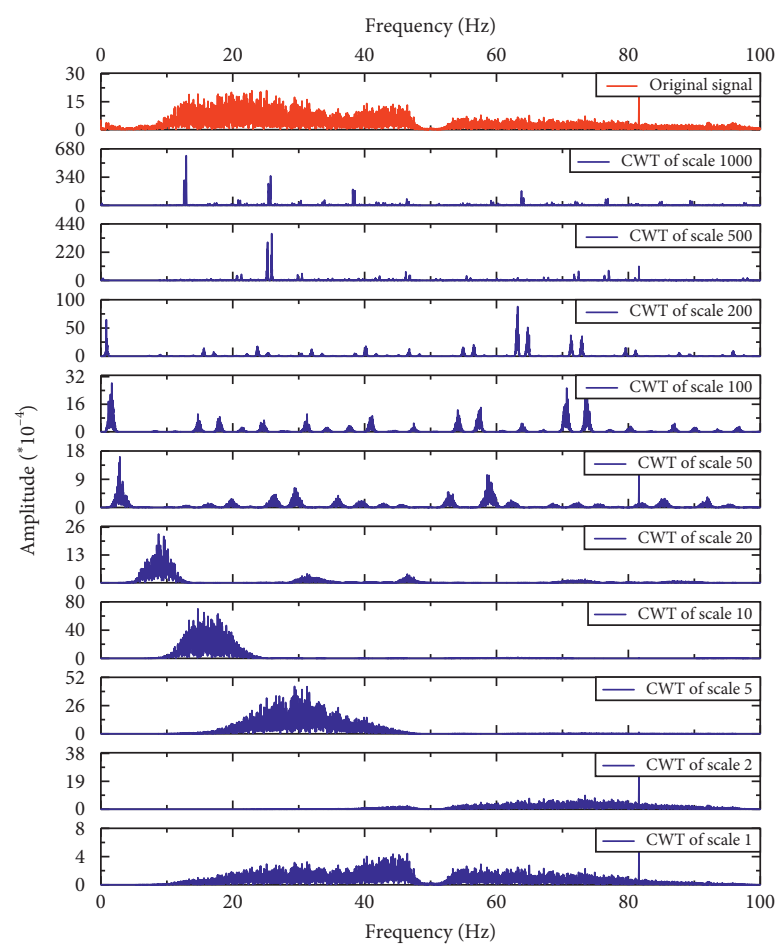

(b)

FIgURE 14: CWT analysis of SA5 at the invert of the upper-span tunnel at different scales: (a) time history; (b) Fourier spectrum.

TABLE 6: Peak acceleration and frequency of the invert at the central cross section of the upper-span tunnel.

\begin{tabular}{|c|c|c|c|c|c|c|}
\hline Transformation scale & $\begin{array}{c}\text { Peak acceleration } \\
\left(\mathrm{m} / \mathrm{s}^{2}\right)\end{array}$ & $R_{a i}$ & $\begin{array}{l}\text { Corresponding } \\
\text { time }(\mathrm{s})\end{array}$ & $\begin{array}{c}\text { Main frequency } \\
\text { band }(\mathrm{Hz})\end{array}$ & Dominant frequency $(\mathrm{Hz})$ & $R_{f i}$ \\
\hline Original signal & 0.23 & - & 40.56 & $5-48$ & 26 & - \\
\hline 1 & 0.05 & 0.22 & 51.25 & $10-50$ & 46 & 1.77 \\
\hline 2 & 0.11 & 0.48 & 53.56 & $52-83$ & 74 & 2.92 \\
\hline 5 & 0.28 & 1.22 & 51.26 & $14-47$ & 32 & 1.23 \\
\hline 10 & 0.30 & 1.30 & 51.72 & $18-30$ & 15 & 0.57 \\
\hline 20 & 0.11 & 0.22 & 48.47 & $7-13$ & 9 & 0.35 \\
\hline 50 & 0.05 & 0.48 & 48.51 & $3-6$ & 5 & 0.19 \\
\hline
\end{tabular}

$$
\begin{aligned}
& R_{a i}=\frac{a_{i}}{a_{o}} \\
& R_{f i}=\frac{f_{i}}{f_{o}},
\end{aligned}
$$

where $a_{0}$ represents the peak acceleration of the original signal, $a_{i}$ represents the peak acceleration of the transformation-scale signal, $f_{o}$ represents the dominant frequency of the original signal, $f_{i}$ represents the dominant frequency of the transformation-scale signal, and $i=1,2,5,10,20$, and 50 .

Figure 15 presents the time-transformation scalecoefficient matrix and frequency-transformation scaleamplitude matrix. For $R_{a i}$ and $R_{f i}$, changes mainly appeared at a low transformation scale. With an increase in the transformation scale, the peak value of acceleration first increased and then decreased. When the transformation scale was 10 , the peak value was maximized $\left(0.30 \mathrm{~m} / \mathrm{s}^{2}\right)$. The corresponding frequency $R_{f i}$ growth law was similar to that of the acceleration, which first increased and then decreased with an increase in the transformation scale. When the transformation scale was between 5 and 10, the peak acceleration and dominant frequency were closest to the original signal. These different responses indicate that, at the transformation of small scales, the medium and low frequencies play a leading role in the invert of the orthogonal upper-span overlapped tunnel. Thus, the medium- and low-frequency responses of the upper-span tunnel under seismic loading are stronger than highfrequency response. Additionally, the absorption effect of the surrounding rock around the tunnel for high-frequency seismic waves is significant [50, 51]. Furthermore, the resonance between the low- and medium-frequency seismic waves and the tunnel structure should be avoided, so as to prevent the deformation and damage of the tunnel when the earthquake disaster is aggravated in the engineering application. 


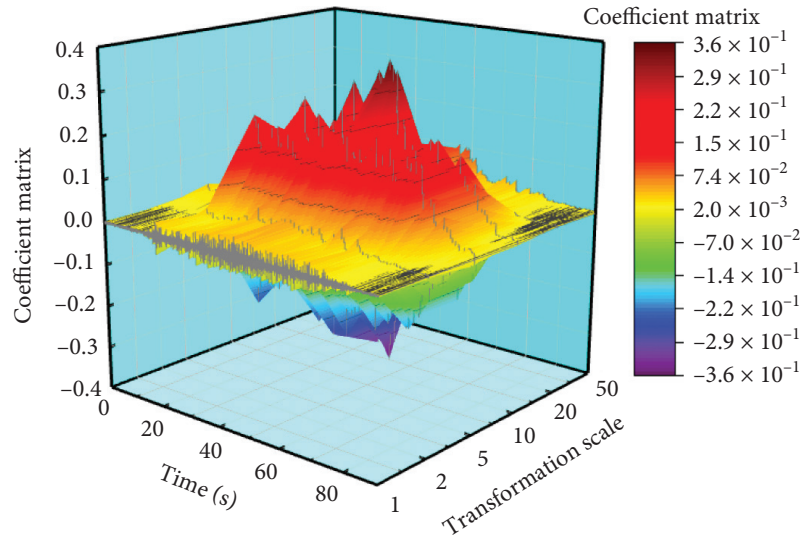

(a)

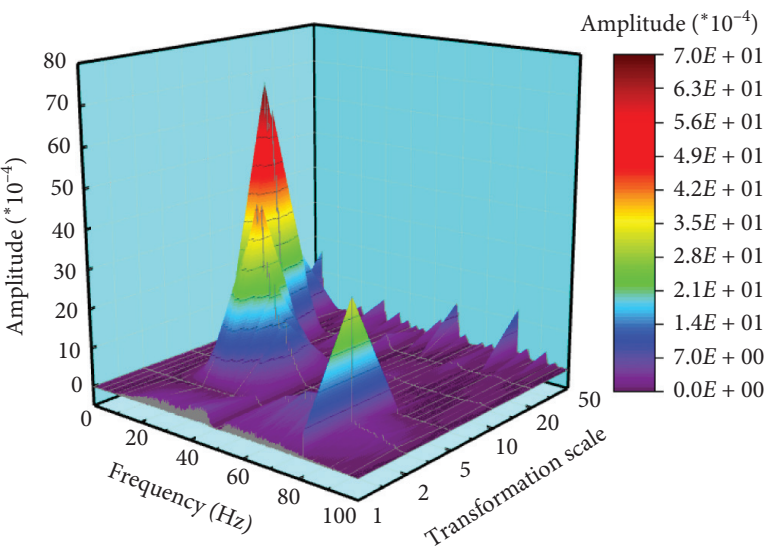

(b)

FIGURE 15: Three-dimensional (3D) color mapping curve for the invert of the upper-span tunnel: (a) time-transformation scale-coefficient matrix; (b) frequency-transformation scale-amplitude.

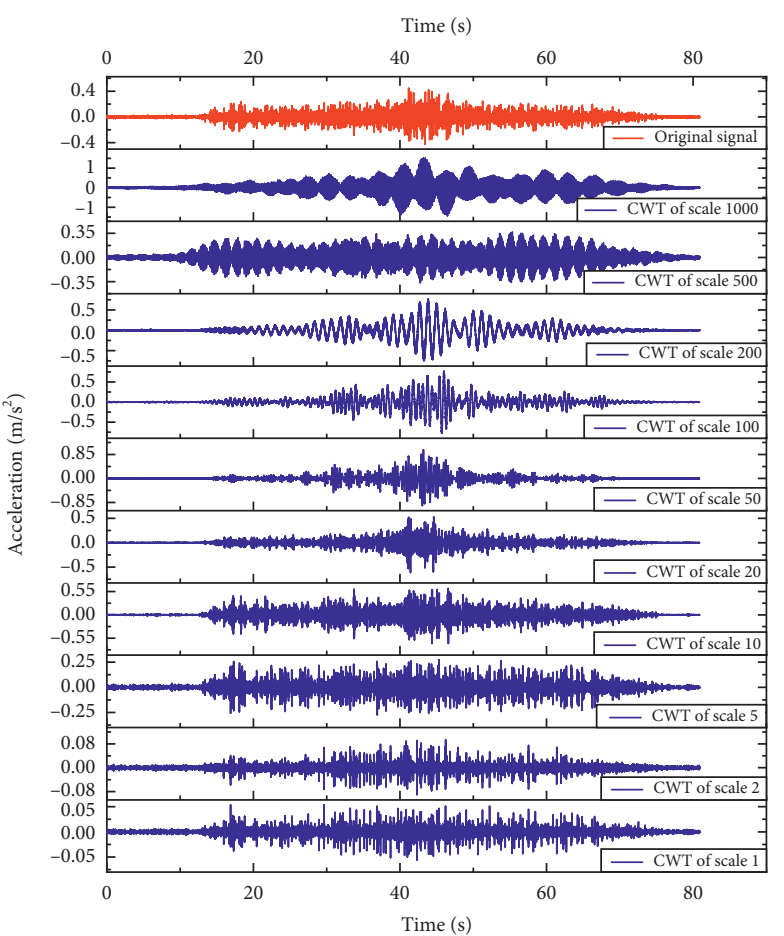

(a)

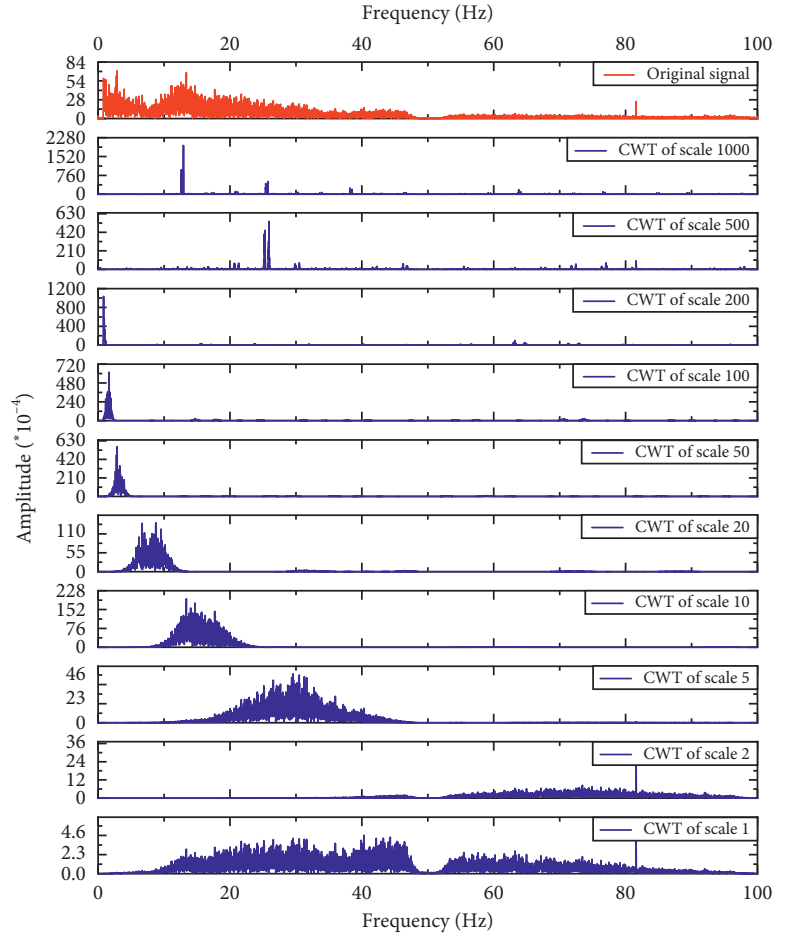

(b)

FIGURE 16: CWT analysis of XA2 at the crown of the under-crossing tunnel at different scales: (a) time history; (b) Fourier spectrum.

TABLE 7: Peak acceleration and frequency of the crown at the central cross section of the under-crossing tunnel.

\begin{tabular}{lccccc}
\hline Transformation scale & Peak acceleration $\left(\mathrm{m} / \mathrm{s}^{2}\right)$ & $R_{a i}$ & $\begin{array}{c}\text { Corresponding } \\
\text { time }(\mathrm{s})\end{array}$ & $\begin{array}{c}\text { Main frequency } \\
\text { band }(\mathrm{Hz})\end{array}$ & $\begin{array}{c}\text { Dominant } \\
\text { frequency }(\mathrm{Hz})\end{array}$ \\
\hline Original signal & 0.49 & - & 43.90 & $2-46$ & - \\
$R_{f i}$
\end{tabular}




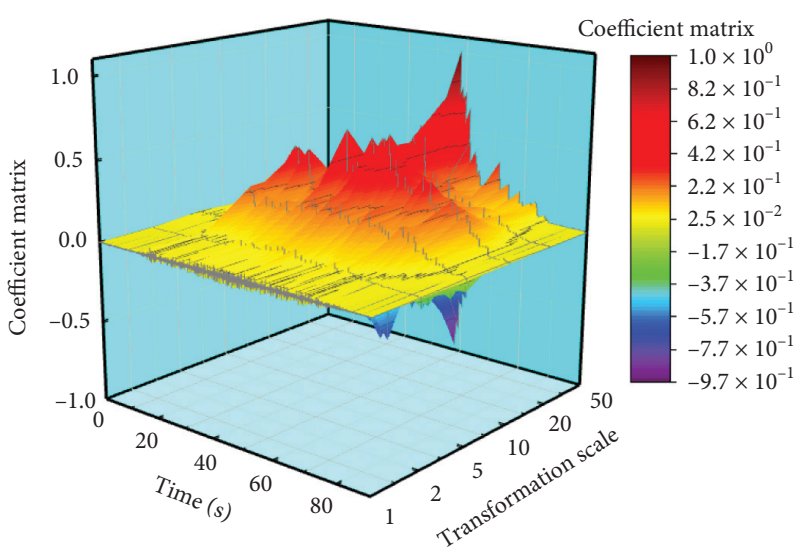

(a)

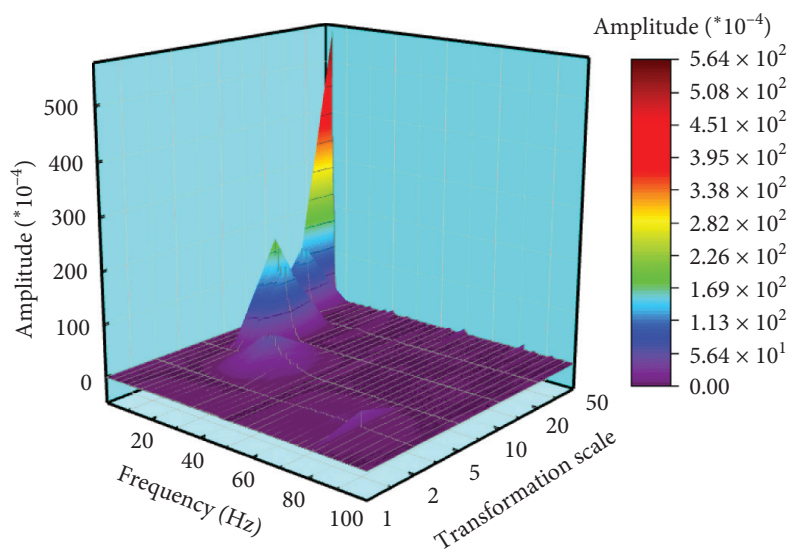

(b)

FIGURE 17: 3D color mapping curve for the crown of the under-crossing tunnel: (a) time-transformation scale-coefficient matrix; (b) frequency-transformation scale-amplitude.

4.3. CWT of the Crown (XA2) of Under-Crossing Tunnel. Figure 16 shows the typical time history and Fourier spectrum of the crown of the under-crossing tunnel transformed by XA2. For the acceleration dynamic response of the XA2 measurement point, the response of the transformation of small scales was larger in the frequency band of $0-50 \mathrm{~Hz}$ of the original signal. Synchronously, the acceleration response was relatively strong within 25-50 s after loading. $R_{a i}$ and $R_{f i}$ of the CWT at the transformation of small scales $(1,2,5,10$, and 50$)$ were also analyzed, as shown in Table 7.

Similarly, the time-transformation scale-coefficient matrix and frequency-transformation scale-amplitude are shown in Figures 17(a) and 17(b), respectively. As for $R_{a i}$ and $R_{f i}$, distinct changes mainly appeared at a low transformation scale. With an increase in the transformation scale, $R_{a i}$ first increased and then decreased. When the transformation scale was 50 , the peak value was maximized $\left(0.94 \mathrm{~m} / \mathrm{s}^{2}\right)$. The growth law of the frequency $R_{f i}$ was slightly different from that of the acceleration, which first increased and then decreased with the increase in the transformation scale. Remarkably, the peak acceleration and dominant frequency were closest to the original signal when the transformation scale was between 10 and 20 . The results of these analyses suggest that, at the transformation of small scales, the medium and low frequencies play an important role in the crown of the orthogonal under-crossing overlapped tunnel.

The peak acceleration and frequency characteristics of the invert of the upper-span tunnel and the crown of the under-crossing tunnel were compared at the cross section, respectively. Overall, the seismic response at the crown of the under-crossing tunnel was stronger than that at the invert of the upper-span tunnel. According to the study of single tunnel by Zhao et al. [52], the dynamic response of tunnel decreased with the increase of depths. However, the results of this study showed that the dynamic response of the under-crossing tunnel invert decreased, while the crown increased instead of decreasing. This indicates that there was a superposition effect on the acceleration response of the crown of the under-crossing tunnel owing to the existence of the upper-span tunnel. Additionally, the acceleration response was relatively intense within 25-60 s after the seismic wave was inputted; thus, attention should be paid to protecting the overlapped tunnel in this process. Analysis of the corresponding spectrum characteristics revealed that the medium and low frequencies under the transformation of small scales (5-20) played a dominant role in the overlapped tunnel under seismic loading.

\section{Conclusions}

The seismic response of an orthogonal overlapped tunnel under horizontal seismic loading was investigated through a large-scale shaking table test with a geometric similarity ratio of $1: 50$. A mixture of cement, coarse sand, soil, and water was used to simulate the surrounding rock. The lining structure of the tunnel was made of a mixture of gypsum, quartz sand, and water. Different input accelerations were consecutively applied to the shaking table in the horizontal direction. The distributions of the peak accelerations of the crown and invert in the orthogonal overlapped tunnel were studied separately. Moreover, the spectral characteristics of the invert of the upper-span tunnel and the crown of the under-crossing tunnel at the cross section were analyzed using the Morlet wavelet of the CWT. According to the results, the following conclusions are drawn:

(1) For the upper-span tunnel, the failure of the tunnel basically evolved from the right to the cross section and then to the left side, and acceleration response showed obvious "surface effect" in the horizontal direction with increase of maximum input acceleration. For the under-crossing tunnel, the peak acceleration of the crown and invert exhibited "parabolic" and "V" shape distribution, respectively. Additionally, owing to the existence of the upperspan tunnel and the spatial effect, the seismic 
response of the invert at the cross section of the under-crossing tunnel was significantly weakened.

(2) The model exhibited obvious deformation stages during the seismic wave loading process, which can be divided into elastic, plastic, plastic enhancement, and failure stage. When the seismic intensity was high, the acceleration amplification factor had a jump increase, and the seismic response growth trend of the central section was significantly greater than that of both affected sides, indicating that the cross section was an obvious weak area, which was easy to cause the damage of tunnels.

(3) The seismic response at the crown of the undercrossing tunnel was stronger than that at the invert of the upper-span tunnel, because of "superposition effect." Additionally, the acceleration response was strongest within 25-60 s after the seismic wave was inputted. Therefore, it is necessary to pay attention to the antiearthquake design of the invert of the upperspan tunnel and the crown of the under-crossing tunnel for overlapped tunnel.

(4) An analysis of the corresponding spectrum characteristics revealed that the medium and low frequencies in the transformation of small scales (5-20) played a dominant role in the overlapped tunnel under seismic loading, while the surrounding rock around the tunnel had an obvious absorption effect on high frequency. It is necessary to avoid the resonance of low-medium frequency seismic waves with the tunnel structure to prevent the deformation and damage of the tunnel during the earthquake disaster.

\section{Data Availability}

The data used to support the findings of this study are available from the corresponding author upon request.

\section{Conflicts of Interest}

The authors declare that they have no conflicts of interest.

\section{Authors' Contributions}

Hao Lei contributed to this work.

\section{Acknowledgments}

This research was supported by the National Key R\&D Program of China (2018YFC1504903) and the Project on Science and Technology Development Project of China Railway Nine Bureau Group Co., Ltd., Dalian Branch (KJ-2019-01). The authors gratefully acknowledge the support from the Key Laboratory of Loess Earthquake Engineering, CEA.

\section{References}

[1] Q. Fang, D. Zhang, Q. Li, and L. N. Y. Wong, "Effects of twin tunnels construction beneath existing shield-driven twin tunnels," Tunnelling and Underground Space Technology, vol. 45, pp. 128-137, 2015.

[2] H. Mohamad, P. J. Bennett, K. Soga, R. J. Mair, and K. Bowers, "Behaviour of an old masonry tunnel due to tunnellinginduced ground settlement," Geotechnique, vol. 60, no. 12, pp. 927-938, 2010.

[3] J. R. Standing and R. Selman, "The response to tunnelling of existing tunnels at Waterloo and Westminster," Journal of Biomolecular Structure and Dynamics, vol. 31, no. 2, pp. 6-7, 2001.

[4] T. Balendra, D. P. Thambiratnam, C. G. Koh, and S.-L. Lee, "Dynamic response of twin circular tunnels due to incident SH-waves," Earthquake Engineering and Structural Dynamics, vol. 12, no. 2, pp. 181-201, 1984.

[5] V. Besharat, M. Davoodi, and M. K. Jafari, "Effect of underground structures on free-field ground motion during earthquakes," in Proceedings of the 15th World Conference on Earthquake Engineering, Lisbon, Portugal, September 2012.

[6] M. Panji and B. Ansari, "Anti-plane seismic ground motion above twin horseshoe-shaped lined tunnels," Innovative Infrastructure Solutions, vol. 5, no. 1, pp. 1-16, 2020.

[7] C. M. St. John and T. F. Zahrah, "Aseismic design of underground structures," Tunnelling and Underground Space Technology, vol. 2, no. 2, pp. 165-197, 1987.

[8] T. Grigorios, D. S. Filomena, A. Ioannis et al., "Seismic behaviour of tunnels: from experiments to analysis," Tunnelling and Underground Space Technology, vol. 90, Article ID 103334, 2020.

[9] Y. M. A. Hashash, J. J. Hook, B. Schmidt, and J. I. C. Yao, "Seismic design and analysis of underground structures," Tunnelling and Underground Space Technology, vol. 16, no. 2, pp. 247-293, 2001.

[10] Z. Zhang, M. Huang, C. Xu, Y. Jiang, and W. Wang, "Simplified solution for tunnel-soil-pile interaction in Pasternak's foundation model," Tunnelling and Underground Space Technology, vol. 78, no. 8, pp. 146-158, 2018.

[11] Z. Zhang, M. Zhang, Y. Jiang, Q. Bai, and Q. Zhao, “Analytical prediction for ground movements and liner internal forces induced by shallow tunnels considering non-uniform convergence pattern and ground-liner interaction mechanism," Soils and Foundations, vol. 57, no. 2, pp. 211-226, 2017.

[12] G. D. Hatzigeorgiou and D. E. Beskos, "Soil-structure interaction effects on seismic inelastic analysis of 3-D tunnels," Soil Dynamics and Earthquake Engineering, vol. 30, no. 9, pp. 851-861, 2010.

[13] X. F. Ma, G. B. Wang, J. Wu, and Q. Q. Ji, "Experimental study on the seismic response of subway station in soft ground," Journal of Earthquake and Tsunami, vol. 11, no. 5, Article ID 1750020, 2017.

[14] H. MrouehI. Shahrour et al., "Three-dimensional finite element analysis of the interaction between tunneling and pile foundations," International Journal for Numerical and Analytical Methods in Geomechanics, vol. 26, no. 3, pp. 217-230, 2002.

[15] B. J. Rama and A. Ramanathan, "Interaction of urban underground twin metro tunnels under static and earthquake loading," Journal of Earthquake and Tsunami, vol. 14, no. 4, Article ID 2050019, 2020.

[16] L. Jiang, J. Chen, and J. Li, "Seismic response of underground utility tunnels: shaking table testing and FEM analysis," Earthquake Engineering and Engineering Vibration, vol. 9, no. 4, pp. 555-567, 2010.

[17] G. C. Wang, J. Yao, L. Sha, L. C. Yu, and X. W. Zheng, "Analysis on the seismic response of hangzhou metro tunnel 
in soft soils," Advanced Materials Research, vol. 446-449, no. 446-449, pp. 966-969, 2012.

[18] J. X. Lai, K. Y. Wang, J. L. Qiu et al., "Vibration response characteristics of the cross tunnel structure," Shock and Vibration, vol. 2016, Article ID 9524206, 16 pages, 2016.

[19] H. Yu, Y. Yuan, G. Xu, Q. Su, X. Yan, and C. Li, "Multi-point shaking table test for long tunnels subjected to non-uniform seismic loadings - part II: application to the HZM immersed tunnel," Soil Dynamics and Earthquake Engineering, vol. 108, pp. 187-195, 2018.

[20] F. Wang, X. Jiang, and J. Niu, "The large-scale shaking table model test of the shallow-bias tunnel with a small clear distance," Geotechnical and Geological Engineering, vol. 35, no. 3, pp. 1093-1110, 2017.

[21] X. Jiang, F. Wang, H. Yang, G. Sun, and J. Niu, "Dynamic response of shallow-buried small spacing tunnel with asymmetrical pressure: shaking table testing and numerical simulation," Geotechnical and Geological Engineering, vol. 36, no. 4, pp. 2037-2055, 2018.

[22] G. Chen, Z. Wang, X. Zuo, X. Du, and H. Gao, "Shaking table test on the seismic failure characteristics of a subway station structure on liquefiable ground," Earthquake Engineering and Structural Dynamics, vol. 42, no. 10, pp. 1489-1507, 2013.

[23] D. Zhao, J. Shen, G. Chen, and J. Zhu, "Shaking table experimental study on time frequency characteristics of subway stations constructed in different materials," in Proceedings of the GSIC 2018: Proceedings of Geo Shanghai 2018 International Conference: Advances in Soil Dynamics and Foundation Engineering, Shanghai, China, May 2018.

[24] Y. Kawamata, M. Nakayama, I. Towhata, and S. Yasuda, "Dynamic behaviors of underground structures in E-Defense shaking experiments," Soil Dynamics and Earthquake Engineering, vol. 82, pp. 24-39, 2016.

[25] G. Wang, M. Yuan, Y. Miao, J. Wu, and Y. Wang, "Experimental study on seismic response of underground tunnel-soilsurface structure interaction system," Tunnelling and Underground Space Technology, vol. 76, pp. 145-159, 2018.

[26] M. B. MasoudRabeti and H. B. Mohammad, "Seismic ground motion amplification pattern induced by a subway tunnel: shaking table testing and numerical simulation," Soil Dynamics and Earthquake Engineering, vol. 83, pp. 81-97, 2016.

[27] C. Liu, L.-M. Peng, M.-F. Lei, and Y.-F. Li, "Research on crossing tunnels' seismic response characteristics," KSCE Journal of Civil Engineering, vol. 23, no. 11, pp. 4910-4920, 2019.

[28] Ö. Aydan, Y. Ohta, M. Geniş, N. Tokashiki, and K. Ohkubo, "Response and stability of underground structures in rock mass during earthquakes," Rock Mechanics and Rock Engineering, vol. 43, no. 6, pp. 857-875, 2010.

[29] R. E. S. Moss and V. A. Crosariol, "Scale model shake table testing of an underground tunnel cross section in soft clay," Earthquake Spectra, vol. 29, no. 4, pp. 1413-1440, 2013.

[30] Z. Bao, Y. Yuan, and H. Yu, "Multi-scale physical model of shield tunnels applied in shaking table test," Soil Dynamics and Earthquake Engineering, vol. 100, pp. 465-479, 2017.

[31] H. Xu, T. Li, L. Xia, J. X. Zhao, and D. Wang, "Shaking table tests on seismic measures of a model mountain tunnel," Tunnelling and Underground Space Technology, vol. 60, pp. 197-209, 2016.

[32] N. Ma, H. Wu, H. Ma, X. Wu, and G. Wang, "Examining dynamic soil pressures and the effectiveness of different pile structures inside reinforced slopes using shaking table tests," Soil Dynamics and Earthquake Engineering, vol. 116, pp. 293-303, 2019.
[33] X. Zuo, G. X. Chen, and H. Y. Zhuang, "Comparison between circular and straight-wall-top-arch tunnels under seismic response based on numerical simulation," Journal of Disaster Prevention and Mitigation Engineering, vol. 27, no. 4, pp. 401-406, 2007.

[34] Z. Guan, Y. Zhou, X. Gou, H. Huang, and X. Wu, "The seismic responses and seismic properties of large section mountain tunnel based on shaking table tests," Tunnelling and Underground Space Technology, vol. 90, pp. 383-393, 2019.

[35] J. X. Lai, S. Y. He, J. L. Qiu et al., "Characteristics of seismic disasters and aseismic measures of tunnels in Wenchuan earthquake," Environmental Earth Sciences, vol. 76, p. 94, 2017.

[36] Y. Q. Chen, X. L. Lv, and W. Huang, "Simulation method of soil boundary conditions in shaking table test of structurefoundation interaction," Structural Engineer, no. 3, pp. 25-30, 2000, in Chinese.

[37] CY. Wu, J. H. Zong, Y. T. Li et al., "Scheme design of shaking table model test of tunnel-soil-superior building interaction system," Journal of Hebei University of Technology, vol. 48, no. 6, pp. 61-68, 2019, in Chinese.

[38] B. W. Xu and X. L. Jiang, "Soil chamber design of shaking test table for large-scale soil-pile-complex structure interaction," Journal of Tianjin University, vol. 43, no. 10, p. 912, 2010, in Chinese.

[39] Y. Q. Chen, X. L. Lv, P. Z. Li, and B. Chen, "Comparative study on shaking table model test of foundation-structure dynamic interaction with different soil properties," China Civil Engineering Journal, no. 5, pp. 57-64, 2006, in Chinese.

[40] J.-P. Antoine, P. Carrette, R. Murenzi, and B. Piette, "Image analysis with two-dimensional continuous wavelet transform," Signal Processing, vol. 31, no. 3, pp. 241-272, 1993.

[41] C. D. Saragiotis, L. J. Hadjileontiadis, and S. M. Panas, "A higher-order statistics-based phase identification of threecomponent seismograms in a redundant wavelet transform domain," in Proceedings of the IEEE Signal Processing Workshop on Higher-Order Statistics, pp. 396-399, Madison, Wisconsin, June 1999.

[42] A. Grinsted, J. C. Moore, and S. Jevrejeva, "Application of the cross wavelet transform and wavelet coherence to geophysical time series," Nonlinear Processes in Geophysics, vol. 11, no. 5/6, pp. 561-566, 2004.

[43] K. Bauer, B. Norden, A. Ivanova, M. Stiller, and C. M. Krawczyk, "Wavelet transform-based seismic facies classification and modelling: application to a geothermal target horizon in the NE German Basin," Geophysical Prospecting, vol. 68, no. 2, pp. 466-482, 2019.

[44] O. O. Lukianchenko, Y. V. Vorona, and O. E. Kostina, "Wavelet analysis of seismic wave reaction of frame building," Strength of Materials and Theory of Structures, vol. 103, pp. 131-144, 2019.

[45] P. J. McGetrick and C. W. Kim, "A parametric study of a drive by bridge inspection system based on the morlet wavelet," Key Engineering Materials, vol. 569-570, pp. 262-269, 2013.

[46] O. Yazdanpanah, B. Mohebi, and M. Yakhchalian, "Selection of optimal wavelet-based damage-sensitive feature for seismic damage diagnosis," Measurement, vol. 154, p. 107447, 2020.

[47] I. De Moortel, S. A. Munday, and A. W. Hood, "Wavelet analysis: the effect of varying basic wavelet parameters," Solar Physics, vol. 222, no. 2, pp. 203-228, 2004.

[48] T. Kijewski and A. Kareem, "Wavelet transforms for system identification in civil engineering," Computer-Aided Civil and Infrastructure Engineering, vol. 18, no. 5, pp. 339-355, 2003. 
[49] J. Y. Yin, Y. Q. Zhu, Z. P. Song et al., "Significance test and precision analysis of Morlet wavelet: application to studying periodic variation of earthquake activity and geomagnetic field," Acta Seismologica Sinica, vol. 33, no. 5, pp. 663-671, 2011.

[50] N. N. Liu, Q. B. Huang, Y. J. Ma et al., "Experimental study of a segmented metro tunnel in a ground fissure area," Soil Dynamics and Earthquake Engineering, vol. 100, pp. 410-416, 2011.

[51] J. Zhang, Y. Yuan, Z. Bao, H. Yu, and E. Bilotta, "Shaking table tests on the intersection of cross passage and twin tunnels," Soil Dynamics and Earthquake Engineering, vol. 124, pp. 136-150, 2019.

[52] X. Zhao, R. H. Li, M. Zhao, and L. J. Tao, "Shaking table model tests on tunnels at different depths," in Proceedings of the Ghent, BELGIUM: 6th International Symposium on Life-Cycle Civil Engineering, Ghent, Belgium, October 2018. 